\title{
Existence, nonexistence, and asymptotic behavior of solutions for $N$-Laplacian equations involving critical exponential growth in the whole $\mathbb{R}^{N}$
}

\author{
Anderson L. A. de Araujo ${ }^{1}$ and Luiz F. O. Faria ${ }^{2}$ \\ ${ }^{1}$ Universidade Federal de Viçosa, Departamento de Matemática, \\ Av. Peter Henry Rolfs, s/n, Viçosa, MG, Brazil, CEP 36570-900., \\ E-mail: anderson.araujo@ufv.br \\ ${ }^{2}$ Universidade Federal de Juiz de Fora, ICE, Departamento de Matemática, \\ Rua José Lourenço Kelmer, s/n Juiz de Fora, MG, Brazil, CEP 36036-900, \\ E-mail: luiz.faria@ufjf.edu.br
}

\begin{abstract}
In this paper, we are interested in studying the existence or nonexistence of solutions for a class of elliptic problems involving the $N$ Laplacian operator in the whole space. The nonlinearity considered involves critical Trudinger-Moser growth. Our approach is non-variational, and in this way we can address a wide range of problems not yet contained in the literature. Even $W^{1, N}\left(\mathbb{R}^{N}\right) \hookrightarrow L^{\infty}\left(\mathbb{R}^{N}\right)$ failing, we establish $\left\|u_{\lambda}\right\|_{L^{\infty}\left(\mathbb{R}^{N}\right)} \leq C\|u\|_{W^{1, N}\left(\mathbb{R}^{N}\right)}^{\Theta}$ (for some $\Theta>0$ ), when $u$ is a solution. To conclude, we explore some asymptotic properties.
\end{abstract}

Keywords: Dirichlet problem; Galerkin approximation; Critical growth; MoserTrudinger inequality; $N$-Laplacian.

2020 MSC: 35A01; 35A25; 35B09; 35B20; 35B33; 35B40; 35J92.

\section{Introduction}

Let $W_{0}^{1, p}(\Omega), \Omega \subseteq \mathbb{R}^{N}, N \geq 2$, be the Sobolev space endowed with the norm

$$
\|u\|_{W^{1, p}(\Omega)}=\left(\int_{\Omega}\left(|\nabla u|^{p}+|u|^{p}\right) d x\right)^{\frac{1}{p}}
$$

If $p<N$, the critical growth $p^{*}=N p /(N-p)$ means that $\|u\|_{W_{0}^{1, p}(\Omega)} \hookrightarrow L^{q}(\Omega)$, $N \leq p \leq p^{*}$. The case $p=N$ is a borderline case in the sense of Sobolev embeddings. As is well-known, one has $\|u\|_{W_{0}^{1, N}(\Omega)} \hookrightarrow L^{q}(\Omega), q \geq N$, but a function in $W_{0}^{1, N}(\Omega)$ may have a local singularity and this causes the failure of the embedding $W^{1, N}(\Omega) \not \subset L^{\infty}(\Omega)$. If $\Omega$ is a bounded domain, another kind of 
maximal growth was proved by Yudovič [38], Pohožaev [35] and Trudinger [36]. They proved, in an independent way, that

$$
u \in W_{0}^{1, N}(\Omega) \Longrightarrow \int_{\Omega} e^{|u|^{N^{\prime}}} d x<\infty,
$$

where $N^{\prime}=\frac{N}{N-1}$. Moreover, for any higher growth, the corresponding integral can be infinite for a suitable choice of $u$. After that, Moser [30] improved this assertion, showing that if $u \in W_{0}^{1, N}(\Omega)$, then

$$
\sup _{\|\nabla u\|_{L^{N}(\Omega)} \leq 1} \int_{\Omega} e^{\alpha|u|^{N^{\prime}}} d x \begin{cases}\leq c|\Omega|, & \text { if } \alpha \leq \alpha_{N} \\ =\infty, & \text { if } \alpha>\alpha_{N}\end{cases}
$$

where $\alpha_{N}=N \omega_{N-1}^{1 /(N-1)}, c$ is a constant which depends on $N$, and $\omega_{N-1}$ is the measure of the unit sphere in $\mathbb{R}^{N}$. Inequality (2) is now called Moser-Trudinger inequality and the term $e^{\alpha_{N}|u|^{N^{\prime}}}$ is known as critical Moser-Trudinger growth.

This well-known Moser-Trundinger inequality has been generalized in many ways. In the case $\Omega=\mathbb{R}^{N}$, B. Ruf when $N=2$ in [33], and Y.X. Li and B. Ruf in [25] for $N>2$, proved the following assertion

$$
\sup _{\|u\|_{W^{1, N}\left(\mathbb{R}^{N}\right)} \leq 1} \int_{\mathbb{R}^{N}} \phi_{N}\left(\alpha|u|^{\frac{N}{N-1}}\right) d x \begin{cases}\leq C(\alpha, N)^{N^{\prime}}, & \text { if } \alpha \leq \alpha_{N}, \\ =\infty, & \text { if } \alpha>\alpha_{N},\end{cases}
$$

where $\alpha_{N}>0$ is a constant and

$$
\phi_{N}(t)=e^{t}-\sum_{j=0}^{N-2} \frac{t^{j}}{j !}=\sum_{j=N-1}^{\infty} \frac{t^{j}}{j !}, t \geq 0 .
$$

Remark 1. Notice that $\phi_{N}(t)$ is a increasing function.

In this paper, we consider the existence, nonexistence, and asymptotic behavior of positive solutions for a class of $N$-Laplacian elliptic equations related to the critical growth (3) in the whole space $\mathbb{R}^{N}$. More precisely, we are concerned with the following problem:

$$
-\Delta_{N} u+|u|^{N-2} u=\lambda a(x)|u|^{q-2} u+f(u) \text { in } \mathbb{R}^{N},
$$

where $\lambda>0,2 \leq N, 1<q<N, a$ is a positive function such that $a \in$ $L^{\frac{N}{N-q}}\left(\mathbb{R}^{N}\right) \cap L^{\infty}\left(\mathbb{R}^{N}\right), f: \mathbb{R} \rightarrow \mathbb{R}$ is a continuous function satisfying the growth condition

$$
0 \leq f(t) t \leq a_{1}|t|^{p} \phi_{N}\left(\alpha|t|^{\frac{N}{N-1}}\right), t \in \mathbb{R},
$$

where $N<p<+\infty$, and $\alpha>0$.

In this setting, we mean by a solution of problem (5) any function $u \in$ $W^{1, N}\left(\mathbb{R}^{N}\right) \cap C_{\text {loc }}^{1}\left(\mathbb{R}^{N}\right)$, such that

$$
\int_{\mathbb{R}^{N}}\left(|\nabla u|^{N-2} \nabla u \nabla \phi+|u|^{N-2} u \phi-\lambda a(x)|u|^{q-2} u \phi-f(u) \phi\right) d x=0,
$$


for all $\phi \in C_{0}^{\infty}\left(\mathbb{R}^{N}\right)$.

Besides existence, this paper is regarding explore some asymptotic properties of the obtained solutions. If $u_{\lambda}$ is a solution of (5), we are interested in investigating the following properties:

$$
\begin{gathered}
u_{\lambda}(x) \rightarrow 0 \text { as }|x| \rightarrow \infty, \\
\left\|u_{\lambda}\right\|_{W^{1, N}\left(\mathbb{R}^{N}\right)} \rightarrow 0 \text { as } \lambda \rightarrow 0^{+},
\end{gathered}
$$

and

$$
\left\|u_{\lambda}\right\|_{L^{\infty}\left(\mathbb{R}^{N}\right)} \rightarrow 0 \text { as } \lambda \rightarrow 0^{+} .
$$

Furthermore, concerning to problem (5), we scrutinize the nonexistence of solution for $\lambda$ large enough.

The class of equations in (5) appears as a model for several problems in the fields of electromagnetism, astronomy, and fluid dynamics. They can be used to accurately describe the behavior of electric, gravitational, and fluid potentials. Problems of the form (5) are important in several applications to the study of evolution equations of $N$-laplacian type that appear in non-Newtonian fluids, turbulent flows in porus media and other contexts.

The case $N=2$ is also related to the stationary problem associated with the following initial value Schrödinger equation

$$
i \partial_{t} u+\Delta u+\mu g(u)=0, \quad \text { in } \mathbb{R}_{t} \times \mathbb{R}_{x}^{2},
$$

with data

$$
u_{0}:=u(0, \cdot) \in W^{1,2}\left(\mathbb{R}^{2}\right),
$$

where $u:=u(t, x)$ is a complex-valued function of $(t, x) \in \mathbb{R} \times \mathbb{R}^{2}$, and

$$
g(u):=u\left(e^{4 \pi|u|^{2}}-1\right) .
$$

Schrödinger equations involving exponential nonlinearities have several applications, such as the self-trapped beans in plasma [23]. In [7], the author considered Schrödinger equation with decreasing exponential nonlinearity. The stationary problem associated to (10) is given by

$$
\Delta \phi-\phi+g(\phi)=0, \quad \phi \in W^{1,2}\left(\mathbb{R}^{2}\right) .
$$

If $\phi \in W^{1,2}\left(\mathbb{R}^{2}\right)$ is a solution to (13), then $e^{i t} \phi$ is a solution to (10) called a soliton or a standing wave. Equations of type (13) arise in various other contexts of physics such that, the classical approximation in statistical mechanics, constructive field theory, false vacuum in cosmology, nonlinear optics, laser propagations, etc. They are also called nonlinear Euclidean scalar field equations, see $[3,8,16,18]$.

Recently, there has been considerable interest in the study of existence results for problems of the form

$$
\left\{\begin{array}{lll}
-\Delta_{N} u+V(x)|u|^{N-2} u=g(x, u) & \text { in } \mathbb{R}^{N} \\
u>0 & \text { on } \mathbb{R}^{N}
\end{array}\right.
$$


where $g(x, u)$ is continuous and behaves like $\phi_{N}\left(\alpha|u|^{\frac{N}{N-1}}\right)$ as $|u| \rightarrow+\infty$, we would like to mention $[4,11-13,29]$.

In general, the potential $V: \mathbb{R}^{N} \rightarrow \mathbb{R}$ is bounded away from zero, i.e.

$$
V(x) \geq c>0 \quad x \in \mathbb{R}^{N} .
$$

If $V$ is large at infinity in some suitable sense, then the loss of compactness due to the unboundedness of the domain $\mathbb{R}^{N}$ can be overcome and vanishing phenomena can be ruled out. So, a natural framework for the function space setting of problem (14) is given by the subspace $E$ of $W^{1, N}\left(\mathbb{R}^{N}\right)$ defined as

$$
E:=\left\{u \in W^{1, N}\left(\mathbb{R}^{N}\right): \int_{\mathbb{R}^{N}} V(x)|u|^{N} d x<+\infty\right\}
$$

endowed with the norm

$$
\|u\|_{E}:=\left(\int_{\mathbb{R}^{N}}\left(|\nabla u|^{N}+V(x)|u|^{N}\right) d x\right)^{\frac{1}{N}} \forall u \in E .
$$

Under appropriate assumptions on the potential $V$, the embedding

$$
E \hookrightarrow L^{p}\left(\mathbb{R}^{N}\right)
$$

turns out to be compact. For instance, if

$$
V^{-1} \in L^{\frac{1}{N-1}}\left(\mathbb{R}^{N}\right),
$$

then the embedding (15) is compact for any $p \geq 1$ (see e.g. [37, Lemma 2.4]), while assuming the weaker condition

$$
V^{-1} \in L^{1}\left(\mathbb{R}^{N}\right)
$$

the embedding (15) becomes compact only for $p \geq N$ (see [9], see also [14] for other compactness results).

The authors of $[12,13,24,37]$, considering a potential $V$ satisfying (16) or (17), obtained existence results for equations of the form (14) and even more general equations. Their proofs rely, crucially, on the compact embeddings (15), given by (16) and (17) (in particular, on the compact embedding of $E$ into $\left.L^{N}\left(\mathbb{R}^{N}\right)\right)$.

Most papers treat problem (14) employing variational methods. Then, usually, it is assumed that $g$ has subcritical or critical growth and sometimes asking

$$
g(s) \geq c|s|^{p-1}, \text { for each } s \geq 0 \text { where } c>0 \text { and } p>N
$$

are constants, see $[2,11]$.

Another common assumption on $g$ is the so-called Ambrosetti-Rabinowitz condition, that is,

$\exists R>0$ and $\theta>N$ such that $0<\theta G(x, s) \leq s g(x, s) \forall|s| \geq R$ and $x \in \mathbb{R}^{N}$, 
where $G(s)=\int_{0}^{s} g(t) d t$, see [11, 12, 24, 29].

Even when the Ambrosetti-Rabinowitz is dropped, it is usually to be assumed some additional condition to obtain compactness of the Palais-Samle sequences or Cerami sequences. See, for instance, [2, 24], where the authors assume, respectively, the set of conditions

$$
\left.\begin{array}{r}
\exists t_{0}>0 \text { and } M>0 \text { such that } 0<G(x, s) \leq M g(x, s), \\
\forall|s| \geq t_{0} \text { and } x \in \Omega ; \\
0<N G(x, s) \leq s g(x, s) \forall|s| \geq 0 \text { and } x \in \Omega,
\end{array}\right\}
$$

and

$$
\left.\begin{array}{r}
H(x, t) \leqslant H(x, s) \text { for all } 0<t<s, \forall x \in \mathbb{R}^{N}, \\
\text { where } H(x, u)=u f(x, u)-N F(x, u) .
\end{array}\right\}
$$

The case when the potential $V$ is constant, i.e. $V(x)=c$ for any $x \in \mathbb{R}^{N}$, is much less developed. In such a case, only a few existence results are known, and we should like to mention [29] (and references therein), where the authors proved existence results by means Variational approach. In [11], the author consider $V(x)=1$ and $g(x, t)=\lambda h(x)|t|^{q-2} t+f(t), x \in \mathbb{R}^{N}$, as in (6), with $f \in C^{1}(\mathbb{R}, \mathbb{R})$ satisfying the Ambrosetti-Rabinowitz condition.

In the following, we state our main results.

Theorem 1.1. Suppose that $f: \mathbb{R} \rightarrow \mathbb{R}$ is a continuous function satisfying assumption (6). Then there exists $\lambda^{*}>0$ such that for every $\lambda \in\left(0, \lambda^{*}\right)$ problem (5) admits a positive solution $u_{\lambda} \in W^{1, N}\left(\mathbb{R}^{N}\right) \cap C_{\text {loc }}^{1}\left(\mathbb{R}^{N}\right)$. Furthermore,

$$
\left\|u_{\lambda}\right\|_{W^{1, N}\left(\mathbb{R}^{N}\right)} \rightarrow 0
$$

as $\lambda \rightarrow 0^{+}$.

Proposition 1. Suppose that $f(t)=|t|^{p-1} \phi_{N}\left(\alpha|t|^{\frac{N}{N-1}}\right)$ and let

$$
\lambda^{*}=\sup \left\{\lambda>0 ;(5) \text { has a solution } u_{\lambda} \text { in } W^{1, N}\left(\mathbb{R}^{N}\right)\right\} .
$$

Then $\lambda^{*}<\infty$.

Theorem 1.2. Suppose that $f: \mathbb{R} \rightarrow \mathbb{R}$ is a continuous function satisfying assumption (6). Then any solution $u_{\lambda}, \lambda \in\left(0, \lambda^{*}\right)$, given by Theorem 1.1, satisfies $u_{\lambda}(x) \rightarrow 0$ as $|x| \rightarrow \infty$.

Corollary 1. Any solution $u_{\lambda}, \lambda \in\left(0, \lambda^{*}\right)$, given by Theorem 1.1, satisfies

$$
\left\|u_{\lambda}\right\|_{L^{\infty}\left(\mathbb{R}^{N}\right)} \rightarrow 0
$$

as $\lambda \rightarrow 0^{+}$.

Notice that in this paper we don not impose any extra hypotheses on $f$ beyond (6). To compare our condition on the nonlinearity of problem (5) with we have found in the literature, we present two examples that follow. 
Example 1. As the first example of a function satisfying (6), we have

$$
f(t):=h(t) \phi_{N}\left(\alpha|t|^{\frac{N}{N-1}}\right)
$$

where

$$
h(t)=|t|^{p-2} t \sin ^{2}(t) .
$$

Now, define

$$
g(x, t):=\lambda a(x) t^{q-1}+h(t) \phi_{N}\left(\alpha|t|^{\frac{N}{N-1}}\right),
$$

where $a \in L^{\frac{N}{N-q}}\left(\mathbb{R}^{N}\right) \cap L^{\infty}\left(\mathbb{R}^{N}\right)$ is a positive function. Notice that (22) satisfies neither (18) nor Ambrosetti-Rabinowitz condition nor (20). First, let us verify that $g$ does not satisfy (18). Let $c>0$ be a positive fixed constant. Taking the sequence $t_{k}=2 k \pi$, with $k$ a positive integer, $q<N$ and $a \in L^{\infty}\left(\mathbb{R}^{N}\right)$, there holds $g\left(x, t_{k}\right)=\lambda a(x) t_{k}^{q-1}<c t_{k}^{p-1}$, for $k$ large enough.

Now, let us define

$$
\begin{aligned}
H(x, t)= & t\left[\lambda a(x) t^{q-1}+|t|^{p-2} t \sin ^{2}(t) \phi_{N}\left(\alpha|t|^{\frac{N}{N-1}}\right)\right] \\
& -N \int_{0}^{t}\left(\lambda a(x) s^{q-1}+|s|^{p-2} s \sin ^{2}(s) \phi_{N}\left(\alpha|s|^{\frac{N}{N-1}}\right)\right) d s .
\end{aligned}
$$

Still considering the sequence $t_{k}=2 k \pi$, with $k$ a positive integer, $H$ satisfies

$$
H\left(x, t_{k}\right)=\lambda a(x) t_{k}^{q}\left(1-\frac{N}{q}\right)-N \int_{0}^{t_{k}}|s|^{p-2} s \sin ^{2}(s) \phi_{N}\left(\alpha|s|^{\frac{N}{N-1}}\right) d s<0 .
$$

Notice that $H\left(x, t_{k}\right)>H\left(x, t_{k+1}\right)$. Therefore, (22) satisfies neither AmbrosettiRabinowitz condition nor (19) nor (20).

Example 2. The second example of a function satisfying (6) we would like to mention is

$$
f(t):=|t|^{p-2} t\left[(\sin (t))_{+}\right] \phi_{N}\left(\alpha|t|^{\frac{N}{N-1}}\right), \text { where } z_{+}=\max \{z, 0\} .
$$

Notice that $f$ is a continuous function but it is not derivable. Indeed, since

$$
\begin{aligned}
f_{-}^{\prime}(\pi) & =\lim _{l \rightarrow 0^{-}} \frac{f(\pi+l)}{l}=\lim _{l \rightarrow 0^{-}} \frac{|\pi+l|^{p-2}(\pi+l) \sin (\pi+l) \phi_{N}\left(\alpha|\pi+l|^{\frac{N}{N-1}}\right)}{l} \\
& =-\lim _{l \rightarrow 0^{-}}|\pi+l|^{p-2}(\pi+l) \frac{\sin (l)}{l} \phi_{N}\left(\alpha|\pi+l|^{\frac{N}{N-1}}\right) \\
& =-\pi^{p-1} \phi_{N}\left(\alpha \pi^{\frac{N}{N-1}}\right)<0,
\end{aligned}
$$

and

$$
f_{+}^{\prime}(\pi)=\lim _{l \rightarrow 0^{+}} \frac{f(\pi+l)}{l}=0,
$$

so $f^{\prime}(\pi)$ does not exists. In particular, $f$ does not belong to $C^{1}(\mathbb{R}, \mathbb{R})$, as it imposed in [11] to obtain the existence result. Furthermore, if $N \geq 2$, (23) satisfies neither Ambrosetti-Rabinowitz condition nor (20). Indeed, let us define

$$
\begin{aligned}
\tilde{H}(t)= & t\left(|t|^{p-2} t\left[(\sin (t))_{+}\right] \phi_{N}\left(\alpha|t|^{\frac{N}{N-1}}\right)\right) \\
& -N \int_{0}^{t}|s|^{p-2} s\left[(\sin (s))_{+}\right] \phi_{N}\left(\alpha|s|^{\frac{N}{N-1}}\right) d s .
\end{aligned}
$$


Considering the sequence $t_{k}=2 k \pi$, with $k$ being a positive integer, $\tilde{H}$ satisfies

$$
\tilde{H}\left(t_{k}\right)=-N \int_{0}^{t_{k}}|s|^{p-2} s\left[(\sin (s))_{+}\right] \phi_{N}\left(\alpha|s|^{\frac{N}{N-1}}\right) d s<0 .
$$

Notice that $\tilde{H}\left(t_{k}\right)>\tilde{H}\left(t_{k+1}\right)$. Therefore, (23) does not satisfies neither AmbrosettiRabinowitz condition nor (19) nor (20).

By observing these simple examples above, we can see that our results are not included in the previous literature. Therefore, it brings novelty in the study of such equations in the field.

The solution of (5) is obtained as the limit of auxiliarily problems in bounded domains. The technique combines the Galerkin method, comparison principle of lower and upper solutions, and regularity scheme. As in [10], we also consider a special class of normed spaces of finite dimension. However, to clarify this approach we show Lemma 2.3, which is a result of independent interest. Such a lemma, together Brouwer's fixed point theorem, allows us, in the scheme of the Galerkin method, to work in general Banach spaces of finite dimensions with general norms.

Due to the presence of the critical term $\phi_{N}\left(\alpha|u|^{N-1}\right)$ and since we are in the whole space $\mathbb{R}^{N}$, we had to overcome several difficulties. One of them is a suitable improvement needed in the estimates of the approximating functions of $f$ by comparing it with [10, Lemma 2.2.] (see Lemma 3.2 bellow). Another delicate point in our approach is the regularity needed to ensure that the solution, obtained in the limit, does not vanish. In the bounded domain, we obtain regularity up to the boundary for the auxiliary problems considering approximate functions in the sense of Strauss [34]. For the domain-wide solution case, a kind of a priori estimate in the sup norm is presented, see Proposition 4 (and the consequences of that outcome).

Now we proceed to introduce the organization of the rest of the paper. Section 2 presents comparison principles, some preliminaries results that are useful through the text and an important result labeled as Lemma 2.3. Section 3 is concerning approximating functions that enable us to obtain regularity up to the boundary to the approximating solutions. In Section 4, we study a class of auxiliary problems in bounded domains. Section 5 is devoted to the proof of our main results.

\section{Preliminaries}

In this section, we state some preliminaries results which will be necessary throughout the paper.

Let $u \in W_{0}^{1, N}(D)$. In what follows, let us denote by $\tilde{u}$ the canonical extension of $u$ by 0 outside $D$, that is,

$$
\tilde{u}(x)= \begin{cases}u(x) & \text { if } x \in D \\ 0 & \text { if } x \in \mathbb{R}^{N} \backslash D .\end{cases}
$$


It is well known that $u \in W_{0}^{1, N}(D)$ implies $\tilde{u} \in W^{1, N}\left(\mathbb{R}^{N}\right)$ (see e.g. [5, Proposition 9.18]).

The next technical lemma was proved in [1, Lemma 2.3].

Lemma 2.1. Let $\alpha>0$ and $r>1$. Then, for every $\beta>r$, there exists a constant $C:=C(\beta)$ such that

$$
\left(\phi_{N}\left(\alpha|u|^{\frac{N}{N-1}}\right)\right)^{r} \leq C \phi_{N}\left(\beta \alpha|u|^{\frac{N}{N-1}}\right),
$$

where $\phi_{N}$ is given in (4).

A complete proof of the next result can be found in [15, Lemma 3].

Lemma 2.2. Let $1<q<N$. For any constants $b>0$, the problem

$$
\begin{cases}-\Delta_{N} u+|u|^{N-2} u=b|u|^{q-2} u & \text { in } D \\ u>0 & \text { in } D, \\ u=0 & \text { on } \partial D\end{cases}
$$

admits a solution $u_{b} \in C_{0}^{1}(\bar{D})$ satisfying $\partial u_{b} / \partial \nu<0$ on $\partial D$.

We conclude this section by presenting a lemma, which is a consequence of Brouwer's Fixed Point Theorem. However, our statement is a subtle (but very useful) generalization by comparing it with the literature. In particular, this result allows us to work in general Banach spaces, with freedom in choosing the norm (see the proof of Lemma 4.2). We will adopt $|x|_{2}=\sqrt{\langle x, x\rangle}$ to denote the usual euclidean norm in $\mathbb{R}^{d}$ and $\|x\|_{d}$ to denote a general norm in $\mathbb{R}^{d}$. The proof of next lemma follows some ideas like in Kesavan [21], where the result for the particular case $\|x\|_{d}:=|x|_{2}$ is presented.

Lemma 2.3. Let $F:\left(\mathbb{R}^{d},\|\cdot\|_{d}\right) \rightarrow\left(\mathbb{R}^{d},\|\cdot\|_{d}\right)$ be a continuous function such that $\langle F(\xi), \xi\rangle \geq 0$ for every $\xi \in \mathbb{R}^{d}$ with $\|\xi\|_{d}=\varrho$ for some $\varrho>0$, and $\langle\cdot, \cdot\rangle^{1 / 2}=|\cdot|_{2}$. Then, there exists $z_{0}$ in the closed ball $\bar{B}_{\varrho}^{d}(0):=\left\{z \in \mathbb{R}^{d} ;\|z\|_{d} \leq \varrho\right\}$ such that $F\left(z_{0}\right)=0$.

Proof. Firstly, there exists $c(d)>0$ such that

$$
\|x\|_{d} \leq c(d)|x|_{2}, \quad \forall x \in \mathbb{R}^{d} .
$$

Suppose, $F(x) \neq 0$ for all $x \in \bar{B}_{\varrho}^{d}(0)$. Define

$$
g:\left(\mathbb{R}^{d},\|\cdot\|_{d}\right) \rightarrow\left(\mathbb{R}^{d},\|\cdot\|_{d}\right)
$$

by

$$
g(x)=-\frac{\varrho}{\|F(x)\|_{d}} F(x)
$$

which maps $\bar{B}_{\varrho}^{d}(0)$ into itself and is continuous. Hence it has a fixed point $x_{0}$, by Brouwer's Fixed Point Theorem. Since $x_{0}=g\left(x_{0}\right)$, we have $\left\|x_{0}\right\|_{d}=\left\|g\left(x_{0}\right)\right\|_{d}=$ $\varrho>0$. But then by $(26)$

$$
0<\varrho^{2}=\left\|x_{0}\right\|_{d}^{2} \leq c(d)^{2}\left|x_{0}\right|_{2}^{2}=c(d)^{2}\left\langle x_{0}, x_{0}\right\rangle=c(d)^{2}\left\langle x_{0}, g\left(x_{0}\right)\right\rangle
$$




$$
=-c(d)^{2} \frac{\varrho}{\left\|F\left(x_{0}\right)\right\|_{d}}\left\langle x_{0}, F\left(x_{0}\right)\right\rangle \leq 0,
$$

by assumptions, which is a contradiction.

\subsection{Comparison principle}

In this ection, we assume that $D$ is a bounded domain in $\mathbb{R}^{N}$ with $C^{2}$ boundary $\partial D$. In the following, we present a couple of comparison principles for a subsolution and a supersolution of the problem

$$
\begin{cases}-\Delta_{N} u+|u|^{N-2} u=g(u) & \text { in } D \\ u=0 & \text { on } \partial D\end{cases}
$$

where $g: \mathbb{R} \rightarrow \mathbb{R}$ is a continuous function.

We say that $u_{1} \in W^{1, N}(D)$ is a subsolution of problem (27) if $u_{1} \leq 0$ on $\partial D$ and

$$
\int_{D}\left(\left|\nabla u_{1}\right|^{N-2} \nabla u_{1} \nabla \varphi+\left|u_{1}\right|^{N-2} u_{1} \varphi\right) d x \leq \int_{D} g\left(u_{1}\right) \varphi d x
$$

for all $\varphi \in W_{0}^{1, N}(D)$ with $\varphi \geq 0$ in $D$ provided the integral $\int_{D} g\left(u_{1}\right) \varphi d x$ exists. We say that $u_{2} \in W^{1, N}(D)$ is a supersolution of (27) if the reversed inequalities are satisfied with $u_{2}$ in place of $u_{1}$ for all $\varphi \in W_{0}^{1, N}(D)$ with $\varphi \geq 0$ in $D$.

The next comparison results are particular cases of the ones achieved in $[15$, Theorem 3, Theorem 5].

Proposition 2. Let $g: \mathbb{R} \rightarrow \mathbb{R}$ be a continuous function such that $g(t) / t^{N-1}$ is decreasing for $t>0$. Assume that $u_{1}$ and $u_{2}$ are a positive subsolution and a positive supersolution of problem (27), respectively. If $u_{2}(x)>u_{1}(x)=0$ for all $x \in \partial D, u_{i} \in C^{1, \alpha}(\bar{D})$ with some $\alpha \in(0,1), \Delta_{N} u_{i} \in L^{\infty}(D)$, for $i, j=1,2$, then $u_{2} \geq u_{1}$ in $D$.

Whenever $u_{1}$ and $u_{2}$ satisfy the homogeneous Dirichlet boundary condition we can state the following result.

Proposition 3. Let $g: \mathbb{R} \rightarrow \mathbb{R}$ be a continuous function such that $g(t) / t^{N-1}$ is decreasing for $t>0$. Assume that $u_{1}, u_{2} \in C_{0}^{1, \alpha}(\bar{D})$ with some $\alpha \in(0,1)$, are a positive subsolution and a positive supersolution of problem (27), respectively. If $\Delta_{N} u_{i} \in L^{\infty}(D)$, for $i, j=1,2, u_{1} / u_{2} \in L^{\infty}(D)$ and $u_{2} / u_{1} \in L^{\infty}(D)$, then $u_{2} \geq u_{1}$ in $D$. 


\section{Approximating functions}

To prove Theorem 1.1, we approximate $f$ by Lipschitz functions $f_{k}: \mathbb{R} \rightarrow \mathbb{R}$ defined by

$$
f_{k}(s)=\left\{\begin{array}{lcc}
-k\left[G\left(-k-\frac{1}{k}\right)-G(-k)\right], & \text { if } & s \leq-k, \\
-k\left[G\left(s-\frac{1}{k}\right)-G(s)\right], & \text { if } & -k \leq s \leq-\frac{1}{k}, \\
k^{2} s\left[G\left(-\frac{2}{k}\right)-G\left(-\frac{1}{k}\right)\right], & \text { if } & -\frac{1}{k} \leq s \leq 0, \\
k^{2} s\left[G\left(\frac{2}{k}\right)-G\left(\frac{1}{k}\right)\right], & \text { if } & 0 \leq s \leq \frac{1}{k}, \\
k\left[G\left(s+\frac{1}{k}\right)-G(s)\right], & \text { if } & \frac{1}{k} \leq s \leq k, \\
k\left[G\left(k+\frac{1}{k}\right)-G(k)\right], & \text { if } & s \geq k,
\end{array}\right.
$$

where $G(s)=\int_{0}^{s} f(\xi) d \xi$.

The following approximation result was proved in [34].

Lemma 3.1. Let $f: \mathbb{R} \rightarrow \mathbb{R}$ be a continuous function such that $s f(s) \geq 0$ for every $s \in \mathbb{R}$. Then there exists a sequence $f_{k}: \mathbb{R} \rightarrow \mathbb{R}$ of continuous functions satisfying

(i) $s f_{k}(s) \geq 0$ for every $s \in \mathbb{R}$;

(ii) $\forall k \in \mathbb{N} \exists c_{k}>0$ such that $\left|f_{k}(\xi)-f_{k}(\eta)\right| \leq c_{k}|\xi-\eta|$ for every $\xi, \eta \in \mathbb{R}$;

(iii) $f_{k}$ converges uniformly to $f$ in bounded subsets of $\mathbb{R}$.

The sequence $f_{k}$ of the previous lemma has some additional properties presented below. Here, we present a suitable improvement in the estimates of the approximating functions $f_{k}$ by comparing it with [10, Lemma 2.2.].

Lemma 3.2. Let $f: \mathbb{R} \rightarrow \mathbb{R}$ be a continuous function satisfying (6) for every $s \in \mathbb{R}$. Then the sequence $f_{k}$ of Lemma 3.1 satisfies

(i) $\forall k \in \mathbb{N}, 0 \leq s f_{k}(s) \leq C_{1}|s|^{p} \phi_{N}\left(2^{\frac{N}{N-1}} \alpha|s|^{\frac{N}{N-1}}\right)$ for every $|s| \geq \frac{1}{k}$;

(ii) $\forall k \in \mathbb{N}, 0 \leq s f_{k}(s) \leq C_{2} \frac{1}{k^{p-2}}|s|^{2}$ for every $|s| \leq \frac{1}{k}$,

where $C_{1}$ and $C_{2}$ are positive constants independent of $k$.

Proof. Everywhere in this proof, the constant $a_{1}$ is the one of (6).

First step. Suppose that $-k \leq s \leq-\frac{1}{k}$.

By the mean value theorem, there exists $\eta \in\left(s-\frac{1}{k}, s\right)$ such that

$$
f_{k}(s)=-k\left[G\left(s-\frac{1}{k}\right)-G(s)\right]=-k G^{\prime}(\eta)\left(s-\frac{1}{k}-s\right)=f(\eta)
$$

and

$$
s f_{k}(s)=s f(\eta) .
$$


Since $s-\frac{1}{k}<\eta<s<0$ and $f(\eta)<0$, we have $s f(\eta) \leq \eta f(\eta)$. Therefore, by using Remark 1, we obtain

$$
\begin{aligned}
s f_{k}(s) \leq \eta f(\eta) & \leq a_{1}|\eta|^{p} \phi_{N}\left(\alpha|\eta|^{\frac{N}{N-1}}\right) \\
& \leq a_{1}\left|s-\frac{1}{k}\right|^{p} \phi_{N}\left(\alpha\left|s-\frac{1}{k}\right|^{\frac{N}{N-1}}\right) \\
& \leq a_{1}\left(|s|+\frac{1}{k}\right)^{p} \phi_{N}\left(\alpha\left(|s|+\frac{1}{k}\right)^{\frac{N}{N-1}}\right) \\
& \leq a_{1}(2|s|)^{p} \phi_{N}\left(\alpha(2|s|)^{\frac{N}{N-1}}\right) \\
& =a_{1} 2^{p}|s|^{p} \phi_{N}\left(2^{\frac{N}{N-1}} \alpha|s|^{\frac{N}{N-1}}\right) .
\end{aligned}
$$

Second step. Assume $\frac{1}{k} \leq s \leq k$.

By the mean value theorem, there exists $\eta \in\left(s, s+\frac{1}{k}\right)$ such that

$$
f_{k}(s)=k\left[G\left(s+\frac{1}{k}\right)-G(s)\right]=k G^{\prime}(\eta)\left(s+\frac{1}{k}-s\right)=f(\eta)
$$

and

$$
s f_{k}(s)=s f(\eta)
$$

Since $0<s<\eta<s+\frac{1}{k}$ and $f(\eta)>0$, we have $s f(\eta) \leq \eta f(\eta)$. Therefore,

$$
\begin{aligned}
s f_{k}(s) \leq \eta f(\eta) & \leq a_{1}|\eta|^{p} \phi_{N}\left(\alpha|\eta|^{\frac{N}{N-1}}\right) \\
& \leq a_{1}\left|s+\frac{1}{k}\right|^{p} \phi_{N}\left(\alpha\left|s+\frac{1}{k_{N}}\right| \frac{N}{N-1}\right) \\
& \leq a_{1}(2|s|)^{p} \phi_{N}\left(\alpha(2|s|)^{\frac{N}{N-1}}\right) \\
& =a_{1} 2^{p}|s|^{p} \phi_{N}\left(2^{\frac{N}{N-1}} \alpha|s|^{\frac{N}{N-1}}\right) .
\end{aligned}
$$

Third step. Suppose that $|s| \geq k$, then

$$
f_{k}(s)= \begin{cases}-k\left[G\left(-k-\frac{1}{k}\right)-G(-k)\right], & \text { if } \quad s \leq-k \\ k\left[G\left(k+\frac{1}{k}\right)-G(k)\right], & \text { if } \quad s \geq k .\end{cases}
$$

If $s \leq-k$, by the mean value theorem, there exists $\eta \in\left(-k-\frac{1}{k},-k\right)$ such that

$$
f_{k}(s)=k\left[G\left(-k-\frac{1}{k}\right)-G(-k)\right]=-k G^{\prime}(\eta)\left(-k-\frac{1}{k}-(-k)\right)=f(\eta)
$$

and

$$
s f_{k}(s)=s f(\eta)
$$


Since $-k-\frac{1}{k}<\eta<-k<0$ and $k<|\eta|<k+\frac{1}{k}$, we conclude that

$$
\begin{aligned}
s f_{k}(s)=\frac{s}{\eta} \eta f(\eta) & \leq \frac{|s|}{|\eta|} a_{1}|\eta|^{p} \phi_{N}\left(\alpha|\eta|^{\frac{N}{N-1}}\right)=a_{1}|s||\eta|^{p-1} \phi_{N}\left(\alpha|\eta|^{\frac{N}{N-1}}\right) \\
& \leq a_{1}|s|\left(k+\frac{1}{k}\right)^{p-1} \phi_{N}\left(\alpha\left(k+\frac{1}{k}\right)^{\frac{N}{N-1}}\right) \\
& \leq a_{1}|s|\left(|s|+\frac{1}{k}\right)^{p-1} \phi_{N}\left(\alpha\left(|s|+\frac{1}{k}\right)^{\frac{N}{N-1}}\right) \\
& \leq a_{1}|s|(2|s|)^{p-1} \phi_{N}\left(\alpha(2|s|)^{\frac{N}{N-1}}\right) \\
& \leq a_{1} 2^{p}|s|^{p} \phi_{N}\left(2^{\frac{N}{N-1}} \alpha|s|^{\frac{N}{N-1}}\right) .
\end{aligned}
$$

If $s \geq k$, by the mean value theorem, there exists $\eta \in\left(k, k+\frac{1}{k}\right)$ such that

$$
f_{k}(s)=k\left[G\left(k+\frac{1}{k}\right)-G(k)\right]=k G^{\prime}(\eta)\left(k+\frac{1}{k}-k\right)=f(\eta) .
$$

By computations similar to conclude (30) one has

$$
\begin{aligned}
& s f_{k}(s)=s f(\eta)=\frac{s}{\eta} \eta f(\eta) \leq \frac{|s|}{|\eta|} a_{1}|\eta|^{p} \phi_{N}\left(\alpha|\eta|^{\frac{N}{N-1}}\right) \leq a_{1} 2^{p}|s|^{p} \phi_{N}\left(2^{\frac{N}{N-1}} \alpha|s|^{\frac{N}{N-1}}\right) . \\
& \text { Fourth step. Assume }-\frac{1}{k} \leq s \leq \frac{1}{k}, \text { then } \\
& \qquad f_{k}(s)= \begin{cases}k^{2} s\left[G\left(-\frac{2}{k}\right)-G\left(-\frac{1}{k}\right)\right], & \text { if } \quad-\frac{1}{k} \leq s \leq 0 \\
k^{2} s\left[G\left(\frac{2}{k}\right)-G\left(\frac{1}{k}\right)\right], & \text { if } \quad 0 \leq s \geq \frac{1}{k} .\end{cases}
\end{aligned}
$$

If $-\frac{1}{k} \leq s \leq 0$, by the mean value theorem, there exists $\eta \in\left(-\frac{2}{k},-\frac{1}{k}\right)$ such that

$$
f_{k}(s)=k^{2} s\left[G\left(-\frac{2}{k}\right)-G\left(-\frac{1}{k}\right)\right]=k^{2} s G^{\prime}(\eta)\left(-\frac{2}{k}-\left(-\frac{1}{k}\right)\right)=-k s f(\eta) .
$$

Therefore

$$
\begin{aligned}
s f_{k}(s) & =-k s^{2} f(\eta)=-k \frac{s^{2}}{\eta} \eta f(\eta) \leq k \frac{s^{2}}{|\eta|} \eta f(\eta) \\
& \leq a_{1} k|s|^{2}|\eta|^{p-1} \phi_{N}\left(\alpha|\eta|^{\frac{N}{N-1}}\right) \leq a_{1} k|s|^{2}\left(\frac{2}{k}\right)^{p-1} \phi_{N}\left(\alpha|\eta|^{\frac{N}{N-1}}\right) \\
& \leq a_{1} 2^{p-1} \frac{|s|^{2}}{k^{p-2}} \phi\left(\alpha\left(\frac{2}{k}\right)^{\frac{N}{N-1}}\right) \\
& \leq a_{1} 2^{p-1} \exp \left(2^{\frac{N}{N-1}} \alpha\right) \frac{1}{k^{p-2}}|s|^{2} .
\end{aligned}
$$

If $0 \leq s \leq \frac{1}{k}$, by the mean value theorem, there exists $\eta \in\left(\frac{1}{k}, \frac{2}{k}\right)$ such that

$$
f_{k}(s)=k^{2} s\left[G\left(\frac{2}{k}\right)-G\left(\frac{1}{k}\right)\right]=k^{2} s G^{\prime}(\eta)\left(\frac{2}{k}-\frac{1}{k}\right)=k s f(\eta) .
$$


By similar computations to conclude (32) one obtains

$$
s f_{k}(s)=k s^{2} f(\eta)=k \frac{s^{2}}{|\eta|} \eta f(\eta) \leq a_{1} 2^{p-1} \exp \left(2^{\frac{N}{N-1}} \alpha\right) \frac{1}{k^{p-2}}|s|^{2} .
$$

The proof of the lemma follows by taking $C_{1}=a_{1} 2^{p}$ ad $C_{2}=a_{1} 2^{p-1} \exp \left(2^{\frac{N}{N-1}} \alpha\right)$, where $a_{1}$ is given in (6).

\section{Solution on bounded domains}

From now on, we assume that $D$ is a bounded domain in $\mathbb{R}^{N}$ with $C^{2}$ boundary $\partial D$. For $r \geq 1$, we denote by $\|u\|_{L^{r}(D)}$ the usual norm on the space $L^{r}(D)$. We endow $W_{0}^{1, N}(D)$ with the norm $\|u\|_{W^{1, N}(D)}^{N}=\|\nabla u\|_{L^{N}(D)}^{N}+\|u\|_{L^{N}(D)}^{N}$.

In this section, we focus on the existence of a positive solution for the problem:

$$
\left\{\begin{array}{lll}
-\Delta_{N} u+|u|^{N-2} u=\lambda a(x)|u|^{q-2} u+f(u) & \text { in } & D, \\
u>0 & \text { in } & D, \\
u(x)=0 & \text { on } & \partial D .
\end{array}\right.
$$

Here $\lambda>0$ is a parameter, $1<q<N, f:[0, \infty) \rightarrow \mathbb{R}$ is a continuous function satisfying (6).

We say that $u \in W_{0}^{1, N}(D)$ is a solution of $(P D)$ if $u(x)>0$ in $D$ and

$$
\int_{D}|\nabla u|^{N-2} \nabla u \nabla \phi d x+\int_{D}|u|^{N-2} u \phi d x=\lambda \int_{D} a(x)|u|^{q-2} u \phi d x+\int_{D} f(u) \phi d x,
$$

for all $\phi \in W_{0}^{1, N}(D)$.

The existence of a solution for problem $(P D)$ is stated below.

Theorem 4.1. Suppose that $f:[0, \infty) \rightarrow \mathbb{R}$ is a continuous function satisfying (6). Then there exists $\lambda^{*}>0$ such that for every $\lambda \in\left(0, \lambda^{*}\right)$ problem $(P D)$ admits a solution $u_{\lambda} \in W_{0}^{1, N}(D)$ such that $\partial u_{\lambda} / \partial \nu<0$ on $\partial D$, where $\nu$ stands for the outer normal to $\partial D$.

\subsection{Approximate equation}

In the proof of Theorem 4.1 we utilize the following auxiliary problem:

$$
\left(P D_{n}\right) \quad \begin{cases}-\Delta_{N} u+|u|^{N-2} u=\lambda a(x)|u|^{q-2} u+f_{n}(u)+\frac{\varphi}{n} & \text { in } D \\ u>0 & \text { in } D \\ u(x)=0 & \text { on } \partial D,\end{cases}
$$

with $n>0$ a integer number, $\varphi$ is a fixed positive function such that $\varphi \in$ $L^{\infty}\left(\mathbb{R}^{N}\right) \cap L^{N^{\prime}}\left(\mathbb{R}^{N}\right)$ and $f_{n}$ is given by Lemma 3.1 and Lemma 3.2. 
Lemma 4.2. There exists $\lambda^{*}>0$ and $n^{*} \in \mathbb{N}$ such that $\left(P D_{n}\right)$ has a solution $u_{n} \in C_{0}^{1}(\bar{D})$ such that $\partial u_{n} / \partial \nu<0$ on $\partial D$ for every $\lambda \in\left(0, \lambda^{*}\right)$ and $n \geq n^{*}$. Furthermore,

$$
\left\|u_{n}\right\|_{W^{1, N}(D)} \leq \varrho,
$$

where @ does not depend on $n$.

Proof. Let $\mathcal{B}=\left\{w_{1}, w_{2}, \ldots, w_{n}, \ldots\right\}$ be a Schauder basis (see $[5,17]$ for details) for the Banach space $\left(W_{0}^{1, N}(D),\|\cdot\|_{W^{1, N}(D)}\right)$. For each positive integer $m$, let

$$
W_{m}=\left[w_{1}, w_{2}, \ldots, w_{m}\right]
$$

be the $m$-dimensional subspace of $W_{0}^{1, N}(D)$ generated by $\left\{w_{1}, w_{2}, \ldots, w_{m}\right\}$ with norm induced from $W_{0}^{1, N}(D)$. Let $\xi=\left(\xi_{1}, \ldots, \xi_{m}\right) \in \mathbb{R}^{m}$, notice that

$$
|\xi|_{m}:=\left\|\sum_{j=1}^{m} \xi_{j} w_{j}\right\|_{W^{1, N}(D)}
$$

defines a norm in $\mathbb{R}^{m}$ (see [10] for the details).

By using the above notation, we can identify the spaces $\left(W_{m},\|\cdot\|_{W^{1, N}(D)}\right)$ and $\left(\mathbb{R}^{m},|\cdot|_{m}\right)$ by the isometric linear transformation

$$
u=\sum_{j=1}^{m} \xi_{j} w_{j} \in W_{m} \mapsto \xi=\left(\xi_{1}, \ldots, \xi_{m}\right) \in \mathbb{R}^{m} .
$$

Now, define the function $F: \mathbb{R}^{m} \rightarrow \mathbb{R}^{m}$ such that

$$
F(\xi)=\left(F_{1}(\xi), F_{2}(\xi), \ldots, F_{m}(\xi)\right),
$$

where $\xi=\left(\xi_{1}, \xi_{2}, \ldots, \xi_{m}\right) \in \mathbb{R}^{m}$,

$$
\begin{aligned}
F_{j}(\xi)= & \int_{D}|\nabla u|^{N-2} \nabla u \nabla w_{j} d x+\int_{D}|u|^{N-2} u w_{j} d x-\lambda \int_{D} a(x)\left(u_{+}\right)^{q-1} w_{j} d x \\
& \quad-\int_{D} f_{n}\left(u_{+}\right) w_{j} d x-\frac{1}{n} \int_{D} \varphi w_{j} d x \\
j=1,2, \ldots, m, \text { and } u= & \sum_{i=1}^{m} \xi_{i} w_{i} \in W_{m} . \text { Therefore } \\
\langle F(\xi), \xi\rangle= & \int_{D}|\nabla u|^{N} d x+\int_{D}|u|^{N} d x-\lambda \int_{D} a(x)\left(u_{+}\right)^{q} d x \\
& -\int_{D} f_{n}\left(u_{+}\right) u_{+} d x-\frac{1}{n} \int_{D} \varphi u d x
\end{aligned}
$$

where $u_{+}=\max \{u, 0\}, u_{-}=u_{+}-u$.

Given $u \in W_{m}$ we define

$$
D_{n}^{+}=\left\{x \in D:|u(x)| \geq \frac{1}{n}\right\}
$$


and

$$
D_{n}^{-}=\left\{x \in D:|u(x)|<\frac{1}{n}\right\} .
$$

Thus, we can write $(35)$ as

$$
\langle F(\xi), \xi\rangle=\langle F(\xi), \xi\rangle_{P}+\langle F(\xi), \xi\rangle_{N},
$$

where

$$
\begin{aligned}
\langle F(\xi), \xi\rangle_{P}= & \int_{D_{n}^{+}}|\nabla u|^{N} d x+\int_{D_{n}^{+}}|u|^{N} d x-\lambda \int_{D_{n}^{+}} a(x)\left(u_{+}\right)^{q} d x \\
& -\int_{D_{n}^{+}} f_{n}\left(u_{+}\right) u_{+} d x-\frac{1}{n} \int_{D_{n}^{+}} \varphi u d x
\end{aligned}
$$

and

$$
\begin{aligned}
\langle F(\xi), \xi\rangle_{N}= & \int_{D_{n^{-}}}|\nabla u|^{N} d x+\int_{D_{n}^{-}}|u|^{N} d x-\lambda \int_{D_{n}^{-}} a(x)\left(u_{+}\right)^{q} d x \\
& -\int_{D_{n}^{-}} f_{n}\left(u_{+}\right) u_{+} d x-\frac{1}{n} \int_{D_{n}^{-}} \varphi u d x .
\end{aligned}
$$

Step 1. In what follows, $C$ denotes a generic real constant. Since the embed$\operatorname{ding} W^{1, N}\left(\mathbb{R}^{N}\right) \subset L^{\tau}\left(\mathbb{R}^{N}\right)$ is continuous for all $\tau \geq N$ (see [5, Corollary 9.11]), we have

$$
\int_{D_{n}^{+}}|a(x)|\left(u_{+}\right)^{q} d x \leq C\|a\|_{L^{N /(N-q)}\left(\mathbb{R}^{N}\right)}\|\tilde{u}\|_{W^{1, N}\left(\mathbb{R}^{N}\right)}^{q} \leq \frac{K_{1}}{2}\|\tilde{u}\|_{W^{1, N}\left(\mathbb{R}^{N}\right)}^{q} .
$$

By virtue of Lemmas 2.1 and 3.2, we get

$$
\begin{aligned}
\int_{D_{n}^{+}} f_{n}\left(u_{+}\right) u_{+} d x & \leq C_{1} \int_{D_{n}^{+}}\left|u_{+}\right|^{p} \phi_{N}\left(2^{\frac{N}{N-1}} \alpha\left|u_{+}\right|^{\frac{N}{N-1}}\right) d x \\
& \leq\left(\int_{\mathbb{R}^{N}}|\tilde{u}|^{N p} d x\right)^{\frac{1}{N}}\left(\int_{\mathbb{R}^{N}}\left(\phi_{N}\left(2^{\frac{N}{N-1}} \alpha|\tilde{u}|^{\frac{N}{N-1}}\right)\right)^{N^{\prime}} d x\right)^{\frac{1}{N^{\prime}}} \\
& \leq K_{2}\|\tilde{u}\|_{W^{1, N}\left(\mathbb{R}^{N}\right)}^{p}\left(\int_{\mathbb{R}^{N}}\left(\phi_{N}\left(N 2^{\frac{2 N}{N-1}} \alpha|\tilde{u}|^{\frac{N}{N-1}}\right)\right) d x\right)^{\frac{1}{N^{\prime}}} \\
& \leq K_{2} C(\alpha, N)\|\tilde{u}\|_{W^{1, N}\left(\mathbb{R}^{N}\right)}^{p}
\end{aligned}
$$

for $\|\tilde{u}\|_{W^{1, N}\left(\mathbb{R}^{N}\right)}$ small enough, where $\tilde{u}$ is given in (24). Indeed, if

$$
\|\tilde{u}\|_{W^{1, N}\left(\mathbb{R}^{N}\right)} \leq \frac{1}{4}\left(\frac{\alpha_{N}}{N \alpha}\right)^{(N-1) / N}
$$

then (3) guarantees the following estimate

$$
\begin{gathered}
\left(\int_{\mathbb{R}^{N}}\left(\phi_{N}\left(N 2^{\frac{2 N}{N-1}} \alpha|\tilde{u}|^{\frac{N}{N-1}}\right)\right) d x\right)^{\frac{1}{N^{\prime}}}= \\
\left(\int_{\mathbb{R}^{N}}\left(\phi_{N}\left(N 2^{\frac{2 N}{N-1}} \alpha\|\tilde{u}\|_{W^{1, N}\left(\mathbb{R}^{N}\right)}^{\frac{N}{N}}\left|\frac{\tilde{u}}{\|\tilde{u}\|_{W^{1, N}\left(\mathbb{R}^{N}\right)}}\right| \frac{N}{N-1}\right)\right) d x\right)^{\frac{1}{N^{\prime}}} \leq C(\alpha, N) .
\end{gathered}
$$


Now, since $\varphi \in L^{N^{\prime}}\left(\mathbb{R}^{N}\right)$, we have

$$
\begin{aligned}
\int_{D_{n}^{+}} \varphi u d x & \leq\|\varphi\|_{L^{N^{\prime}\left(\mathbb{R}^{N}\right)}}\|\tilde{u}\|_{L^{N}\left(\mathbb{R}^{N}\right)} \\
& \leq \frac{K_{3}}{2}\|\tilde{u}\|_{W^{1, N}\left(\mathbb{R}^{N}\right)} .
\end{aligned}
$$

It follows from (36), (37) and (40) that

$$
\begin{aligned}
\langle F(\xi), \xi\rangle_{P} \geq & \int_{D_{n}^{+}}|\nabla u|^{N} d x+\int_{D_{n}^{+}}|u|^{N} d x-\lambda \frac{K_{1}}{2}\|\tilde{u}\|_{W^{1, N}\left(\mathbb{R}^{N}\right)}^{q} \\
& -K_{2} C(\alpha, N)\|\tilde{u}\|_{W^{1, N}\left(\mathbb{R}^{N}\right)}^{p}-\frac{K_{3}}{2 n}\|\tilde{u}\|_{W^{1, N}\left(\mathbb{R}^{N}\right) .}
\end{aligned}
$$

Remark 2. Notice that the constants $K_{1}, K_{2}$ and $K_{3}$ do depend on neither $|D|=\int_{D} d x$ nor $n$.

Step 2. In a similarly way, we obtain

$$
\int_{D_{n}^{-}}|a(x)|\left(u_{+}\right)^{q} d x \leq \frac{K_{1}}{2}\|\tilde{u}\|_{W^{1, N}\left(\mathbb{R}^{N}\right)}^{q} .
$$

By virtue of Lemma 3.2 (ii) we obtain

$$
\begin{aligned}
\int_{D_{n}^{-}} f_{n}\left(u_{+}\right) u_{+} d x & \leq C_{2} \frac{1}{n^{p-2}} \int_{D_{n}^{-}}\left|u_{+}\right|^{2} d x \\
& \leq C_{2} \frac{1}{n^{p-2}}\left(\int_{D_{n}^{-}} d x\right)^{\frac{N-2}{N}}\left(\int_{\mathbb{R}^{N}}|\tilde{u}|^{N} d x\right)^{\frac{2}{N}} \\
& \leq C_{2}|D|^{(N-2) / N} \frac{1}{n^{p-2}}\|\tilde{u}\|_{W^{1, N}\left(\mathbb{R}^{N}\right)}^{2} .
\end{aligned}
$$

We also have

$$
\int_{D_{n}^{-}} \varphi u d x \leq \frac{K_{3}}{2}\|\tilde{u}\|_{W^{1, N}\left(\mathbb{R}^{N}\right)}
$$

It follows from (42), (43) and (44) that

$$
\begin{aligned}
\langle F(\xi), \xi\rangle_{N} \geq & \int_{D_{n}^{-}}|\nabla u|^{N} d x+\int_{D_{n}^{-}}|u|^{N} d x-\lambda \frac{K_{1}}{2}\|\tilde{u}\|_{W^{1, N}\left(\mathbb{R}^{N}\right)}^{q} \\
& -\frac{C_{2}|D|^{(N-2) / N}}{n^{p-2}}\|\tilde{u}\|_{W^{1, N}\left(\mathbb{R}^{N}\right)}^{2}-\frac{K_{3}}{2 n}\|\tilde{u}\|_{W^{1, N}\left(\mathbb{R}^{N}\right)} .
\end{aligned}
$$

Using that $\|u\|_{W^{1, N}(D)}^{N}=\|\tilde{u}\|_{W^{1, N}\left(\mathbb{R}^{N}\right)}^{N}=\|\nabla \tilde{u}\|_{L^{N}\left(\mathbb{R}^{N}\right)}^{N}+\|\tilde{u}\|_{L^{N}\left(\mathbb{R}^{N}\right)}^{N}$, inequalities (41) and (45) imply

$$
\begin{aligned}
\langle F(\xi), \xi\rangle \geq & \|\tilde{u}\|_{W^{1, N}\left(\mathbb{R}^{N}\right)}^{N}-\lambda K_{1}\|\tilde{u}\|_{W^{1, N}\left(\mathbb{R}^{N}\right)}^{q}-K_{2} C(\alpha, N)\|\tilde{u}\|_{W^{1, N}\left(\mathbb{R}^{N}\right)}^{p} \\
& -\frac{C_{2}|D|^{(N-2) / N}}{n^{p-2}}\|\tilde{u}\|_{W^{1, N}\left(\mathbb{R}^{N}\right)}^{2}-\frac{K_{3}}{n}\|\tilde{u}\|_{W^{1, N}\left(\mathbb{R}^{N}\right) .}
\end{aligned}
$$


Now, let $|\xi|_{m}=\|\tilde{u}\|_{W^{1, N}\left(\mathbb{R}^{N}\right)}=\varrho$ for some $\varrho>0$ to be chosen later. Thus, we have

$$
\langle F(\xi), \xi\rangle \geq \varrho^{N}-\lambda K_{1} \varrho^{q}-K_{2} C(\alpha, N) \varrho^{p}-\frac{C_{2}|D|^{(N-2) / N}}{n^{p-2}} \varrho^{2}-\frac{K_{3}}{n} \varrho .
$$

If $\varrho$ is such that

$$
\varrho \leq \frac{1}{\left(2 K_{2} C(\alpha, N)\right)^{\frac{1}{p-N}}}
$$

then

$$
\varrho^{N}-K_{2} C(\alpha, N) \varrho^{p} \geq \frac{\varrho^{N}}{2}
$$

Thus, by choosing

$$
\varrho:=\min \left\{\frac{1}{\left(2 K_{2} C(\alpha, N)\right)^{\frac{1}{p-N}}}, \frac{1}{4}\left(\frac{\alpha_{N}}{N \alpha}\right)^{(N-1) / N}\right\},
$$

we obtain

$$
\langle F(\xi), \xi\rangle \geq \frac{\varrho^{N}}{2}-\lambda K_{1} \varrho^{q}-\frac{C_{2}|D|^{(N-2) / N}}{n^{p-2}} \varrho^{2}-\frac{K_{3}}{n} \varrho .
$$

Now, define $\varsigma:=\frac{\varrho^{N}}{2}-\lambda K_{1} \varrho^{q}$. If we choose

$$
\lambda^{*}:=\frac{\varrho^{N-q}}{4 K_{1}}>0,
$$

then $\varsigma>\frac{\varrho^{N}}{4}$ for all $0<\lambda<\lambda^{*}$. Now, we choose $n^{*} \in \mathbb{N}$ such that

$$
\frac{C_{2}|D|^{(N-2) / N}}{n^{p-2}} \varrho^{2}+\frac{K_{3}}{n} \varrho<\frac{\varsigma}{2},
$$

for every $n \geq n^{*}$. Notice that $n^{*}$ depends on the domain $D$. Since $\xi \in \mathbb{R}^{m}$ is such that $|\xi|_{m}=\varrho$, then for $\lambda<\lambda^{*}$ and $n \geq n^{*}$ we obtain

$$
\langle F(\xi), \xi\rangle \geq \frac{\varsigma}{2}>0
$$

Since $f_{n}$ is a Lipschitz function (for each $n \in \mathbb{N}$ ), it easy to see that $F: \mathbb{R}^{m} \rightarrow$ $\mathbb{R}^{m}$ is a continuous function. Thus, for each $\lambda<\lambda^{*}$ and $n>n^{*}$ fixed, Lemma 2.3 ensure the existence of $y \in \mathbb{R}^{m}$ with $|y|_{m} \leq \varrho$ and such that $F(y)=0$. In other words, there exists $u_{m} \in W_{m}$ verifying

$$
\left\|u_{m}\right\|_{W^{1, N}(D)} \leq \varrho,
$$

and such that

$$
\begin{aligned}
& \int_{D}\left|\nabla u_{m}\right|^{N-2} \nabla u_{m} \nabla w d x+\int_{D}\left|u_{m}\right|^{N-2} u_{m} w d x= \\
& \lambda \int_{D} a(x)\left(u_{m+}\right)^{q-1} w d x+\int_{D} f_{n}\left(u_{m+}\right) w d x+\frac{1}{n} \int_{D} \varphi w d x
\end{aligned}
$$

for all $w \in W_{m}$. 
Remark 3. It is important to mention that $\varrho$, given in (47), does not depend on the domain $D, m$ nor $n$.

Since $W_{m} \subset W_{0}^{1, N}(D) \forall m \in \mathbb{N}$ and $\varrho$ does not depend on $m$, then $\left(u_{m}\right)_{m \in \mathbb{N}}$ is a bounded sequence in $W_{0}^{1, N}(D)$. Therefore, for some subsequence, there exists $u \in W_{0}^{1, N}(D)$ such that

$$
\begin{gathered}
u_{m} \rightarrow u \text { weakly in } W_{0}^{1, N}(D), \\
u_{m} \rightarrow u \text { in } L^{s}(D) s \geq N, \\
u_{m} \rightarrow u, \text { a.e. in } D .
\end{gathered}
$$

Thus,

$$
\|u\|_{W^{1, N}(D)} \leq \liminf _{m \rightarrow \infty}\left\|u_{m}\right\|_{W^{1, N}(D)} \leq \varrho .
$$

We claim that

$$
u_{m} \rightarrow u \text { in } W_{0}^{1, N}(D)
$$

Indeed, using the fact that $\mathcal{B}=\left\{w_{1}, w_{2}, \ldots, w_{n}, \ldots\right\}$ is a Schauder basis of $W_{0}^{1, N}(D)$, for every $u \in W_{0}^{1, N}(D)$ there exists a unique sequence $\left(\alpha_{n}\right)_{n \geq 1}$ in $\mathbb{R}$ such that $u=\sum_{j=1}^{\infty} \alpha_{j} w_{j}$, so that

$$
\psi_{m}:=\sum_{j=1}^{m} \alpha_{j} w_{j} \rightarrow u \text { in } W_{0}^{1, N}(D) \text { as } m \rightarrow \infty
$$

Using $w=\left(u_{m}-\psi_{m}\right) \in W_{m}$ as test function in (50), we obtain

$$
\begin{aligned}
& \int_{D}\left|\nabla u_{m}\right|^{N-2} \nabla u_{m} \nabla\left(u_{m}-\psi_{m}\right) d x+\int_{D}\left|u_{m}\right|^{N-2} u_{m}\left(u_{m}-\psi_{m}\right) d x= \\
& \lambda \int_{D} a(x)\left(u_{m+}\right)^{q-1}\left(u_{m}-\psi_{m}\right) d x+\int_{D} f_{n}\left(u_{m+}\right)\left(u_{m}-\psi_{m}\right) d x \\
& +\frac{1}{n} \int_{D} \varphi\left(u_{m}-\psi_{m}\right) d x .
\end{aligned}
$$

From (51), (52) and (56), it is easy to see that

$$
\begin{aligned}
& \int_{D}\left(\left|u_{m}\right|^{N-1}+\left|\lambda a(x)\left(u_{m+}\right)^{q-1}\right|+\frac{1}{n}|\varphi|\right)\left|u_{m}-\psi_{m}\right| d x \leq \\
& \left(\left\|u_{n}\right\|_{L^{N}(D)}^{N-1}+\lambda\|a\|_{L^{N /(N-q)}(D)}\left\|u_{n}\right\|_{L^{N}(D)}^{q-1}+\frac{1}{n}\|\varphi\|_{L^{N^{\prime}}(D)}\right)\left\|u_{m}-\psi_{m}\right\|_{L^{N}(D)} .
\end{aligned}
$$

By continuity of $f_{n}$ and (53) we obtain

$$
f_{n}\left(u_{m_{+}}\right)^{N^{\prime}} \rightarrow f_{n}\left(u_{+}\right)^{N^{\prime}} \text { a.e. in } D .
$$


By Lemma 3.1, (49), and by using Hölder inequality we obtain

$$
\begin{aligned}
\int_{D} f_{n}\left(u_{m+}\right)^{N^{\prime}} d x & \leq c_{n}^{N^{\prime}} \int_{D}\left|u_{m}\right|^{N^{\prime}} d x \\
& \leq c_{n}^{N^{\prime}} C|D|^{N /\left(N-N^{\prime}\right)}\left\|u_{m}\right\|_{W^{1, N}(D)}^{N^{\prime}} \\
& \leq c_{n}^{N^{\prime}} C|D|^{N /\left(N-N^{\prime}\right)} \varrho^{N^{\prime}} .
\end{aligned}
$$

Hence, [19, Theorem 13.44] leads to

$$
f_{n}\left(u_{m_{+}}\right) \rightarrow f_{n}\left(u_{+}\right) \text {weakly in } L^{N^{\prime}}(D) .
$$

Applying (52), (56) and (60), we conclude that

$$
\lim _{m \rightarrow \infty} \int_{D} f_{n}\left(u_{m+}\right)\left(u_{m}-\psi_{m}\right) d x=0 .
$$

By (49) and (56), we obtain

$$
\lim _{m \rightarrow \infty} \int_{D}\left|\nabla u_{m}\right|^{N-2} \nabla u_{m} \nabla\left(u-\psi_{m}\right) d x=0 .
$$

By (57), (58) (61) and (62), we obtain

$$
\lim _{m \rightarrow \infty} \int_{D}\left|\nabla u_{m}\right|^{N-2} \nabla u_{m} \nabla\left(u_{m}-u\right) d x=0 .
$$

Now it is sufficient to apply the $\left(S_{+}\right)-$property of $-\Delta_{N}$ (see, e.g., [31, Proposition 3.5.]) to obtain (55).

Now, for every $m \geq k$ we obtain

$$
\begin{aligned}
& \int_{D}\left|\nabla u_{m}\right|^{N-2} \nabla u_{m} \nabla w_{k} d x+\int_{D}\left|u_{m}\right|^{N-2} u_{m} w_{k} d x= \\
& \lambda \int_{D} a(x)\left(u_{m+}\right)^{q-1} w_{k} d x+\int_{D} f_{n}\left(u_{m+}\right) w_{k} d x+\frac{1}{n} \int_{D} \varphi w_{k} d x,
\end{aligned}
$$

for all $w_{k} \in W_{k}$.

It follows from (55) and (60) that

$$
\begin{aligned}
& \int_{D}|\nabla u|^{N-2} \nabla u \nabla w_{k} d x+\int_{D}|u|^{N-2} u w_{k} d x= \\
& \lambda \int_{D} a(x)\left(u_{+}\right)^{q-1} w_{k} d x+\int_{D} f_{n}\left(u_{+}\right) w_{k} d x+\frac{1}{n} \int_{D} \varphi w_{k} d x,
\end{aligned}
$$

for all $w_{k} \in W_{k}$. Since $\left[W_{k}\right]_{k \in \mathbb{N}}$ is dense in $W_{0}^{1, N}(D)$ we conclude that

$$
\begin{aligned}
& \int_{D}|\nabla u|^{N-2} \nabla u \nabla w d x+\int_{D}|u|^{N-2} u w d x= \\
& \lambda \int_{D} a(x)\left(u_{+}\right)^{q-1} w d x+\int_{D} f_{n}\left(u_{+}\right) w d x+\frac{1}{n} \int_{D} \varphi w d x
\end{aligned}
$$


for all $w \in W_{0}^{1, N}(D)$. Furthermore, $u \geq 0$ in $D$. In fact, since $u_{-} \in W_{0}^{1, N}(D)$ then from (66) we obtain

$$
\begin{aligned}
-\left\|u_{-}\right\|_{W_{0}^{1, N}(D)}^{N} & =\int_{D}|\nabla u|^{N-2} \nabla u \nabla u_{-} d x+\int_{D}|u|^{N-2} u u_{-} d x \\
& =\lambda \int_{D} a(x)\left(u_{+}\right)^{q-1} u_{-} d x+\int_{D} f_{n}\left(u_{+}\right) u_{-} d x+\frac{1}{n} \int_{D} \varphi u_{-} d x \\
& \geq 0 .
\end{aligned}
$$

Then $u_{-} \equiv 0$ a.e. in $D$, whence $u \geq 0$ a.e. in $D$. Moreover, $u \not \equiv 0$ is valid due to $\frac{\varphi}{n}>0$ in $D$. By applying the strong maximum principle in [32, Theorem 5.4.1] we obtain $u>0$ in $D$, and [32, Theorem 5.5.1] ensure that $\partial u / \partial \nu<0$ on $\partial D$ holds. By Lemma 3.1 and [22, Theorem 7.1] we conclude that $u \in L^{\infty}(D)$. Thus, $[26$, Theorem 1] and [27, p. 320] ensure the regularity up to the boundary $u \in C^{1, \beta}(\bar{D})$, for some $\beta \in(0,1)$.

Therefore, we conclude that proof of the lemma by taking $u_{n}=u$.

\subsection{Proof of Theorem 4.1}

First we show that $(P D)$ has a positive solution. For each $n \in \mathbb{N}, n>n^{*}$, by Lemma 4.2, equation $\left(P D_{n}\right)$ has a solution $u_{n} \in W_{0}^{1, N}(D) \cap C^{1, \beta}(\bar{D})$. Thus

$$
\begin{aligned}
& \int_{D}\left|\nabla u_{n}\right|^{N-2} \nabla u_{n} \nabla w d x+\int_{D}\left|u_{n}\right|^{N-2} u_{n} w d x= \\
& \lambda \int_{D} a(x) u_{n}^{q} w d x+\int_{D} f_{n}\left(u_{n}\right) w d x+\frac{1}{n} \int_{D} \varphi w d x
\end{aligned}
$$

for all $w \in W_{0}^{1, N}(D)$.

By (54) we have that

$$
\left\|u_{n}\right\|_{W^{1, N}(D)} \leq \varrho, \forall n \in \mathbb{N},
$$

and $\varrho$ does not depend on $n$. Thus, along a subsequence again relabeled as $u_{n}$, there exists $u \in W_{0}^{1, N}(D)$ such that

$$
u_{n} \rightarrow u \text { weakly in } W_{0}^{1, N}(D) \text {, as } n \rightarrow \infty .
$$

Thus,

$$
\|u\|_{W^{1, N}(D)} \leq \liminf _{n \rightarrow \infty}\left\|u_{n}\right\|_{W^{1, N}(D)} \leq \varrho
$$

We claim that

$$
u_{n} \rightarrow u W_{0}^{1, N}(D), \text { as } n \rightarrow \infty .
$$

In fact, the proof of (71) follows in a similarly way as we did in the previous section.

First, notice that Lemma 3.1 and (68) imply

$$
\int_{D} f_{n}\left(u_{n}\right)^{N^{\prime}} d x \leq c_{n}^{N^{\prime}} \int_{D}\left|u_{n}\right|^{N^{\prime}} d x \leq c_{n}^{N^{\prime}} C \varrho^{N^{\prime}} .
$$


Moreover,

$$
u_{n} \rightarrow u \text { a.e. in } D,
$$

and by the uniform convergence of Lemma 3.1 (iii) we have

$$
f_{n}\left(u_{n}(\cdot)\right) \rightarrow f(u(\cdot)) \text { a.e. in } D .
$$

Hence, [19, Theorem 13.44] leads to

$$
f_{n}\left(u_{n}\right) \rightarrow f(u) \text { weakly in } L^{N^{\prime}}(D)
$$

On the other hand, taking $w=\left(u_{n}-u\right)$ as a test function in (67), we get

$$
\begin{aligned}
& \int_{D}\left|\nabla u_{n}\right|^{N-2} \nabla u_{n} \nabla\left(u_{n}-u\right) d x \\
\leq & -\int_{D}\left|u_{n}\right|^{N-2} u_{n}\left(u_{n}-u\right) d x+\lambda \int_{D} a(x) u_{n}^{q-1}\left(u_{n}-u\right) d x \\
& +\int_{D} f_{n}\left(u_{n}\right)\left(u_{n}-u\right) d x+\frac{1}{n} \int_{D} \varphi\left(u_{n}-u\right) d x \\
\leq \quad & \left\|u_{n}\right\|_{L^{N}(D)}^{N-1}\left\|u_{n}-u\right\|_{L^{N}(D)} \\
& +\lambda\|a\|_{L^{N /(N-q)}(D)}\left\|u_{n}\right\|_{L^{N}(D)}^{q-1}\left\|u_{n}-u\right\|_{L^{N}(D)} \\
& +\left\|f_{n}\left(u_{n}\right)\right\|_{L^{N^{\prime}}(D)}\left\|u_{n}-u\right\|_{L^{N}(D)} \\
& +\frac{1}{n}\|\varphi\|_{L^{N^{\prime}}(D)}\left\|u_{n}-u\right\|_{L^{N}(D)} \rightarrow 0 \text { as } n \rightarrow \infty .
\end{aligned}
$$

And then, $\limsup _{n \rightarrow \infty} \int_{D}\left|\nabla u_{n}\right|^{N-2} \nabla u_{n} \nabla\left(u_{n}-u\right) d x \leq 0$. Therefore, (71) follows by $\left(S_{+}\right)$property.

By using (71) and (74), we can pass to the limit in (67) to obtain

$$
\int_{D}|\nabla u|^{N-2} \nabla u \nabla w d x+\int_{D}|u|^{N-2} u w d x=\lambda \int_{D} a(x) u^{q-1} w d x+\int_{D} f(u) w d x
$$

for all $w \in W_{0}^{1, N}(D)$. Thus, $u$ is a solution of $(P D)$.

Now, fix a positive constant $\lambda$ such that

$$
\lambda<\lambda^{*}=\frac{\varrho^{N-q}}{4 K_{1}} .
$$

Since $a>0$ is a continuous function, define

$$
a_{D}=\inf _{D} a(x) .
$$

Then, according to Lemma 2.2, there exists a positive solution $u_{0}$ of

$$
\begin{cases}-\Delta_{N} u+|u|^{N-2} u=\lambda a_{D} u^{q-1} & \text { in } D, \\ u>0 & \text { in } D, \\ u=0 & \text { on } \partial D .\end{cases}
$$


Let $u_{n}$ be a positive solution of problem $\left(P D_{n}\right)$ obtained by Lemma 4.2. We observe that $u_{0} / u_{n}, u_{n} / u_{0} \in L^{\infty}(D)$ because $u_{0}$ and $u_{n}$ are positive functions belonging to $C_{0}^{1, \beta}(\bar{D})$ and satisfying $\partial u_{n} / \partial \nu<0, \partial u_{0} / \partial \nu<0$ on $\partial D$. Notice that

$$
\lambda a(x) t^{q-1}+f_{n}(t)+\frac{\varphi}{n} \geq \lambda a_{D} t^{q-1}=g(t) .
$$

Hence, $u_{1}=u_{0}$ and $u_{2}=u_{n}$ are a positive subsolution and a positive supersolution of problem (25), respectively. Thus, by Proposition 3 we see that $u_{n} \geq u_{0}$ in $D$ for every $n>n^{*}$. Therefore, by passing to the limit we obtain

$$
u \geq u_{0} \text { a.e. in } D \text {. }
$$

Thus, we conclude that $u$ is a positive solution of problem $(P D)$.

From now, the solution we just found will be labeled as $u_{\lambda}$ with explicit dependence on $\lambda$. In what follows, we will deduce that $\left\|u_{\lambda}\right\|_{W^{1, N}(D)} \rightarrow 0$ as $\lambda \rightarrow 0$. Fix the pair $\left(\lambda, u_{\lambda}\right)$, where $\lambda \in\left(0, \lambda^{*}\right)$ and $u_{\lambda}$ is the corresponding solution of problem $(P D)$. By using $w=u_{\lambda}$ as a test function in (76), we obtain

$$
\begin{aligned}
\int_{\mathbb{R}^{N}}\left|\nabla \tilde{u}_{\lambda}\right|^{N} d x+\int_{\mathbb{R}^{N}} \tilde{u}_{\lambda}^{N} d x & =\int_{D}\left|\nabla u_{\lambda}\right|^{N} d x+\int_{D} u_{\lambda}^{N} d x \\
& =\lambda \int_{D} a(x) u_{\lambda}^{q} d x+\int_{D} f\left(u_{\lambda}\right) u_{\lambda} d x \\
& =\lambda \int_{\mathbb{R}^{N}} a(x) \tilde{u}_{\lambda}^{q} d x+\int_{\mathbb{R}^{N}} f\left(\tilde{u}_{\lambda}\right) \tilde{u}_{\lambda} d x \\
& \leq \lambda K_{1}\left\|\tilde{u}_{\lambda}\right\|_{W^{1, N}\left(\mathbb{R}^{N}\right)}^{q}+K_{2} C(\alpha, N)\left\|\tilde{u}_{\lambda}\right\|_{W^{1, N}\left(\mathbb{R}^{N}\right)}^{p},
\end{aligned}
$$

where $K_{1}, K_{2}$ are given in (36), (37), respectively.

Since $\tilde{u}_{\lambda} \neq 0$, from (78), we have the following estimate

$$
\left\|\tilde{u}_{\lambda}\right\|_{W^{1, N}\left(\mathbb{R}^{N}\right)}^{N-q}\left(1-K_{2} C(\alpha, N)\left\|\tilde{u}_{\lambda}\right\|_{W^{1, N}\left(\mathbb{R}^{N}\right)}^{p-N}\right) \leq \lambda K_{1} .
$$

By combining (47) and (68), we obtain

$$
\left\|\tilde{u}_{\lambda}\right\|_{W^{1, N}\left(\mathbb{R}^{N}\right)}^{p-N} \leq \frac{1}{2 K_{2} C(\alpha, N)} .
$$

Thus,

$$
\left\|u_{\lambda}\right\|_{W^{1, N}(D)}=\left\|\tilde{u}_{\lambda}\right\|_{W^{1, N}\left(\mathbb{R}^{N}\right)} \leq\left(2 \lambda K_{1}\right)^{1 /(N-q)} \rightarrow 0 \text { as } \lambda \rightarrow 0 .
$$

Thus, the proof of the theorem is complete.

\section{Proof of the Main Theorem}

\subsection{A priori estimates}

In this subsection, for convenience, when necessary, we will omit the notation $\tilde{u}$. In order to prove Theorem 1.1 and 1.2, it will be needed a couple of estimates 
proven in the following results. It is important to mention that much less is known about the results of regularity for the $L_{p}$ operator, as can be seen in [20]. This becomes an obstacle to obtain uniform estimates in the sense of Hölder norm.

Fix $u \in W^{1, N}(D)$ any positive solution of $(P D)$ given by Theorem 4.1. Here, we will borrow some ideas from [15]. Define $u_{M}:=\min \{u, M\}$ for $M>0$. Choose $\bar{p}^{*}$ satisfying $2 N^{2}<\bar{p}^{*}$. For $R^{\prime}>R>0$, we take a smooth function $\eta_{R, R^{\prime}}$ such that $0 \leq \eta_{R, R^{\prime}} \leq 1,\left\|\eta_{R, R^{\prime}}^{\prime}\right\|_{\infty} \leq 2 /\left(R^{\prime}-R\right), \eta_{R, R^{\prime}}(t)=1$ if $t \leq R$ and $\eta_{R, R^{\prime}}=0$ if $t \geq R^{\prime}$.

Lemma 5.1. Let $x_{0} \in \mathbb{R}^{N}, M>0, R^{\prime}>R>0$, such that $B\left(x_{0}, R^{\prime}\right) \subset D$, $\gamma_{1}=\frac{N}{N-q}>1$ and $\gamma_{1}^{\prime}=\frac{N}{q}$ such that $1 / \gamma_{1}+1 / \gamma_{1}^{\prime}=1$. Denote $\eta(x):=$ $\eta_{R, R^{\prime}}\left(\left|x-x_{0}\right|\right)$. Assume that $2 N \leq \tilde{p}$ (in particular $\left.\gamma_{1}^{\prime} \leq \tilde{p}\right)$ and $u \in W^{1, N}(D)$ a solution of $(P D)$, in particular, $u \in L^{\tilde{p}(N+\beta)}\left(B\left(x_{0}, R^{\prime}\right)\right)$ with $\beta \geq 0$. Then it holds:

$$
\begin{gathered}
\int_{B\left(x_{0}, R^{\prime}\right)} f(u) u u_{M}^{\beta} \eta^{N} d x \leq C(\varrho)\|u\|_{L^{\tilde{p}(N+\beta)\left(B\left(x_{0}, R^{\prime}\right)\right)}}^{\beta} B_{R^{\prime}} \\
\int_{B\left(x_{0}, R^{\prime}\right)} a(x) u^{q} u_{M}^{\beta} \eta^{N} d x \leq\|a\|_{L^{\gamma_{1}\left(B\left(x_{0}, R^{\prime}\right)\right)}\|u\|_{L^{\tilde{p}(N+\beta)\left(B\left(x_{0}, R^{\prime}\right)\right)}}^{q+\beta} B_{R^{\prime}}(82)}
\end{gathered}
$$

where $B_{R^{\prime}}:=\left(1+\left|B\left(0, R^{\prime}\right)\right|\right)$ and $\left|B\left(0, R^{\prime}\right)\right|$ denotes the Lebesgue measure of the ball $B\left(0, R^{\prime}\right)$.

Proof. According to Hölder's inequality, we easily show (82). So, we will prove only (81). By Young's inequality, Lemmas 2.1, 3.2, and inequality (38), we obtain

$$
\begin{aligned}
& \int_{B\left(x_{0}, R^{\prime}\right)} f(u) u u_{M}^{\beta} \eta^{N} d x \\
& \leq C \int_{B\left(x_{0}, R^{\prime}\right)}|u|^{p} u_{M}^{\beta} \phi_{N}\left(\alpha|u|^{\frac{N}{N-1}}\right) d x \\
& \leq C\left(\int_{B\left(x_{0}, R^{\prime}\right)}|u|^{N p} u_{M}^{N \beta} d x\right)^{1 / N}\left(\int_{B\left(x_{0}, R^{\prime}\right)}\left[\phi_{N}\left(\alpha|u|^{\frac{N}{N-1}}\right)\right]^{N^{\prime}} d x\right)^{1 / N^{\prime}} \\
& \leq C\left(\int_{B\left(x_{0}, R^{\prime}\right)}|u|^{2 N p} d x\right)^{1 / 2 N}\left(\int_{B\left(x_{0}, R^{\prime}\right)} u_{M}^{2 N \beta} d x\right)^{1 / 2 N} C(\alpha, N) \\
& \leq C\|u\|_{L^{2 N p}\left(B\left(x_{0}, R^{\prime}\right)\right)}^{p}\|u\|_{L^{2 N \beta}\left(B\left(x_{0}, R^{\prime}\right)\right)}^{\beta} \\
& \leq C(\varrho)\|u\|_{L^{2 N \beta}\left(B\left(x_{0}, R^{\prime}\right)\right)}^{\beta},
\end{aligned}
$$

because $\|u\|_{L^{4 p}\left(B\left(x_{0}, R^{\prime}\right)\right)} \leq C\|\tilde{u}\|_{W^{1, N}\left(\mathbb{R}^{N}\right)} \leq C \varrho$.

Since $\tilde{p}(N+\beta)>2 N \beta$, by Hölder's inequality we obtain

$$
\left(\int_{B\left(x_{0}, R^{\prime}\right)} u^{2 N \beta} d x\right)^{1 / 2 N} \leq\|u\|_{L^{\tilde{p}(N+\beta)}\left(B\left(x_{0}, R^{\prime}\right)\right)}^{\beta}\left|B\left(x_{0}, R^{\prime}\right)\right|^{\frac{\tilde{p}(N+\beta)-2 N \beta}{2 N \tilde{p}(N+\beta)}},
$$


that conclude the inequality (81).

Lemma 5.2. Let $x_{0} \in \mathbb{R}^{N}, R^{\prime}>R>0$, such that $B\left(x_{0}, R^{\prime}\right) \subset D, \gamma_{1}=$ $\frac{N}{N-q}>1$ and $\gamma_{1}^{\prime}=\frac{N}{q}$. Assume that $2 N \leq \tilde{p}$. As $u \in L^{\tilde{p}(N+\beta)}\left(B\left(x_{0}, R^{\prime}\right)\right)$ with $\beta \geq 0$, then

$$
\begin{aligned}
& \|u\|_{L}^{N+\beta} \frac{N+\beta}{N}(N+\beta)\left(B\left(x_{0}, R\right)\right) \\
& \quad \leq 2^{N}(N+\beta)^{N} C_{*}^{N} B_{R^{\prime}}\left(C_{R^{\prime}}+D_{R, R^{\prime}}\right) \max \left\{1,\|u\|_{L^{\tilde{p}(N+\beta)}\left(B\left(x_{0}, R^{\prime}\right)\right)}\right\}^{N+\beta}
\end{aligned}
$$

holds with

$$
\begin{aligned}
& B_{R^{\prime}}:=1+\left|B\left(0, R^{\prime}\right)\right| \\
& C_{R^{\prime}}:=C(\varrho)+\lambda^{*}\|a\|_{L^{\gamma_{1}}\left(B\left(x_{0}, R^{\prime}\right)\right)}, \\
& D_{R, R^{\prime}}:=\frac{N^{N} 2^{2 N-1}+2^{N-1}}{\left(R^{\prime}-R\right)^{N}}
\end{aligned}
$$

where $C_{*}$ is the positive constant embedding from $W^{1, N}\left(\mathbb{R}^{N}\right)$ to $L^{\bar{p}^{*}}\left(\mathbb{R}^{N}\right)$.

Proof. Taking $u u_{M}^{\beta} \eta^{N} \in W_{0}^{1, N}\left(B\left(x_{0}, R^{\prime}\right)\right)$ (for $M>0$ ) as test function in (76), where $\eta(x)=\eta_{R, R^{\prime}}\left(\left|x-x_{0}\right|\right)$, and by Lemma 5.1, we obtain

$$
\begin{aligned}
C(\varrho)\|u\|_{L^{\tilde{p}(N+\beta)}\left(B\left(x_{0}, R^{\prime}\right)\right)}^{\beta} B_{R^{\prime}} & \\
& +\lambda\|a\|_{L^{\gamma_{1}}\left(B\left(x_{0}, R^{\prime}\right)\right)}\|u\|_{L^{\tilde{p}(N+\beta)}\left(B\left(x_{0}, R^{\prime}\right)\right)}^{q+\beta} B_{R^{\prime}} \\
\geq & \int_{B\left(x_{0}, R^{\prime}\right)}|\nabla u|^{N} u_{M}^{\beta} \eta^{N} d x+\int_{B\left(x_{0}, R^{\prime}\right)} u_{M}^{N+\beta} \eta^{N} d x \\
& -\frac{2 N}{R^{\prime}-R} \int_{B\left(x_{0}, R^{\prime}\right)}|\nabla u|^{N-1} u_{M}^{\beta} u \eta^{N-1} d x,
\end{aligned}
$$

where we use $|\nabla \eta| \leq 2 /\left(R^{\prime}-R\right)$. From Young's inequality and Hölder's inequality, we obtain

$$
\begin{aligned}
& \frac{2 N}{R^{\prime}-R} \int_{B\left(x_{0}, R^{\prime}\right)}|\nabla u|^{N-1} u_{M}^{\beta} u \eta^{N-1} d x \\
& \quad \leq \frac{1}{2} \int_{B\left(x_{0}, R^{\prime}\right)}|\nabla u|^{N} u_{M}^{\beta} \eta^{N} d x+\frac{2^{N} N^{N} 2^{N-1}}{\left(R^{\prime}-R\right)^{N}} \int_{B\left(x_{0}, R^{\prime}\right)} u^{N+\beta} d x \\
& \quad \leq \frac{1}{2} \int_{B\left(x_{0}, R^{\prime}\right)}|\nabla u|^{N} u_{M}^{\beta} \eta^{N} d x+\frac{N^{N} 2^{2 N-1}}{\left(R^{\prime}-R\right)^{N}}\|u\|_{L^{\tilde{p}(N+\beta)}\left(B\left(x_{0}, R^{\prime}\right)\right)}^{N+\beta} B_{R^{\prime}}(\text {. }
\end{aligned}
$$

Thus, from (84) and (85) we have

$$
\begin{gathered}
B_{R^{\prime}}\left(C_{R^{\prime}}+\frac{N^{N} 2^{2 N-1}}{\left(R^{\prime}-R\right)^{N}}\right) \max \left\{1,\|u\|_{L^{\tilde{p}(N+\beta)}\left(B\left(x_{0}, R^{\prime}\right)\right)}\right\}^{N+\beta} \\
\geq \frac{1}{2} \int_{B\left(x_{0}, R^{\prime}\right)}|\nabla u|^{N} u_{M}^{\beta} \eta^{N} d x+\int_{B\left(x_{0}, R^{\prime}\right)} u_{M}^{N+\beta} \eta^{N} d x
\end{gathered}
$$


Moreover, by using

$$
\begin{aligned}
& \left\|\nabla\left(u_{M}^{1+\beta / N} \eta\right)\right\|_{L^{N}\left(\mathbb{R}^{N}\right)}^{N} \leq 2^{N-1}\left\{\left\|\eta \nabla\left(u_{M}^{1+\beta / N}\right)\right\|_{L^{N}\left(\mathbb{R}^{N}\right)}^{N}+\left\|u_{M}^{1+\beta / N} \nabla \eta\right\|_{L^{N}\left(\mathbb{R}^{N}\right)}^{N}\right\} \\
& \leq 2^{N-1}\left(1+\frac{\beta}{N}\right)^{N} \int_{B\left(x_{0}, R^{\prime}\right)}|\nabla u|^{N} u_{M}^{\beta} \eta^{N} d x+\frac{2^{2 N-1}}{\left(R^{\prime}-R\right)^{N}} \int_{B\left(x_{0}, R^{\prime}\right)} u_{M}^{N+\beta} d x
\end{aligned}
$$

and Hölder's inequality, due to the embedding from $W^{1, N}\left(\mathbb{R}^{N}\right)$ to $L^{\bar{p}^{*}}\left(\mathbb{R}^{N}\right)$, we have

$$
\begin{aligned}
& \frac{1}{2} \int_{B\left(x_{0}, R^{\prime}\right)}|\nabla u|^{N} u_{M}^{\beta} \eta^{N} d x+\int_{B\left(x_{0}, R^{\prime}\right)} u_{M}^{N+\beta} \eta^{N} d x \\
& \geq 2^{-N} N^{N}(N+\beta)^{-N}\left\{\left\|\nabla\left(u_{M}^{1+\beta / N} \eta\right)\right\|_{L^{N}\left(\mathbb{R}^{N}\right)}^{N}+\left\|u_{M}^{1+\beta / N} \eta\right\|_{L^{N}\left(\mathbb{R}^{N}\right)}^{N}\right\} \\
& \quad-\frac{2^{N-1} N^{N}}{(N+\beta)^{N}\left(R^{\prime}-R\right)^{N}} \int_{B\left(x_{0}, R^{\prime}\right)} u_{M}^{N+\beta} d x \\
& \geq 2^{-N} N^{N}(N+\beta)^{-N}\left\|u_{M}^{1+\beta / N} \eta\right\|_{W^{1, N}\left(\mathbb{R}^{N}\right)}^{N} \\
& \quad-\frac{2^{N-1}}{\left(R^{\prime}-R\right)^{N}}\|u\|_{L^{\tilde{p}(N+\beta)}\left(B\left(x_{0}, R^{\prime}\right)\right)}^{N+\beta}\left(1+\left|B\left(0, R^{\prime}\right)\right|\right) \\
& \geq 2^{-N} N^{N}(N+\beta)^{-N} C_{*}^{-N}\left\|u_{M}^{1+\beta / N} \eta\right\|_{L^{\bar{p}^{*}}\left(\mathbb{R}^{N}\right)}^{N} \\
& \quad-\frac{2^{N-1}}{\left(R^{\prime}-R\right)^{N}}\|u\|_{L^{\tilde{p}(N+\beta)}\left(B\left(x_{0}, R^{\prime}\right)\right)}^{N+\beta} B_{R^{\prime}} \\
& \geq 2^{-N} N^{N}(N+\beta)^{-N} C_{*}^{-N}\left\|u_{M}\right\|_{L^{\bar{p}_{*}(N+\beta) / N}\left(B\left(x_{0}, R\right)\right)}^{N+\beta} \\
& \quad-\frac{2^{N-1}}{\left(R^{\prime}-R\right)^{N}}\|u\|_{L^{\tilde{p}(N+\beta)}\left(B\left(x_{0}, R^{\prime}\right)\right)}^{N+\beta} B_{R^{\prime}} .
\end{aligned}
$$

Consequently, it follows from (86) and (87) that

$$
\begin{aligned}
& 2^{-N} N^{N}(N+\beta)^{-N} C_{*}^{-N}\left\|u_{M}\right\|_{L^{\bar{p}^{*}(N+\beta) / N}\left(B\left(x_{0}, R\right)\right)}^{N+\beta} \\
& \quad \leq B_{R^{\prime}}\left(C_{R^{\prime}}+D_{R, R^{\prime}}\right) \max \left\{1,\|u\|_{L^{\tilde{p}(N+\beta)}\left(B\left(x_{0}, R^{\prime}\right)\right)}\right\}^{N+\beta} .
\end{aligned}
$$

The conclusion follows by applying Fatou's lemma and letting $M \rightarrow \infty$ in (88).

Proposition 4. Assume the assumptions of Lemma 5.2. Let us suppose $x_{0} \in D$ and a a positive function such that $a \in L^{\frac{N}{N-q}}\left(\mathbb{R}^{N}\right), f$ satisfying $(6), R_{*}>0$ such that $B\left(x_{0}, 2 R_{*}\right) \subset D$. If $u \in W^{1, N}(D)$ is a solution of $(P D)$, then $u \in L^{\infty}(D)$. Furthermore, $\|u\|_{W^{1, N}(D)} \leq C$ implies $\|u\|_{L^{\infty}(D)} \leq \tilde{C}$. 
Proof. Since $2 N<\bar{p}^{*} / N$, we can choose $\tilde{p}$ such that

$$
2 N<\tilde{p}<\frac{\bar{p}^{*}}{N} .
$$

Let $R_{*}>0$ satisfying $B\left(x_{0}, 2 R_{*}\right) \subset D$. Put

$$
A:=\lambda^{*}\|a\|_{L^{\gamma_{1}\left(\mathbb{R}^{N}\right)}} .
$$

Define sequences $\left\{\beta_{n}\right\},\left\{R_{n}^{\prime}\right\}$ and $\left\{R_{n}\right\}$ by

$$
\begin{gathered}
\beta_{0}:=\frac{\bar{p}^{*}}{\tilde{p}}-N>0, \quad \tilde{p}\left(N+\beta_{n+1}\right)=\frac{\bar{p}^{*}}{N}\left(N+\beta_{n}\right), \\
R_{n}^{\prime}:=\left(1+2^{-n}\right) R_{*}, \quad R_{n}:=R_{n+1}^{\prime} .
\end{gathered}
$$

Since $u \in W^{1, N}(D)$, by using the embedding of $W^{1, N}(D)$ to $L^{\bar{p}^{*}}(D)$, we see that $u \in L^{\bar{p}^{*}}(D)=L^{\tilde{p}\left(N+\beta_{0}\right)}(D)$.

Let $x_{0} \in D$ fixed. Lemma 5.2 guarantees that if $u \in L^{\tilde{p}\left(N+\beta_{n}\right)}\left(B\left(x_{0}, R_{n}^{\prime}\right)\right)$, then $u \in L^{\frac{\bar{T}^{*}}{N}\left(N+\beta_{n}\right)}\left(B\left(x_{0}, R_{n}\right)\right)=L^{\tilde{p}\left(N+\beta_{n+1}\right)}\left(B\left(x_{0}, R_{n+1}^{\prime}\right)\right)$. Notice that

$$
\begin{array}{ll}
B_{R_{n}^{\prime}} & \leq\left(1+\left|B\left(0,2 R_{*}\right)\right|\right)=: B_{0}, \\
C_{R_{n}^{\prime}} & \leq C(\varrho)+A+1=: C_{0}, \\
D_{R_{n}, R_{n}^{\prime}} & =\frac{N^{N} 2^{2 N-1}+2^{N-1}}{R_{*}^{N}} 2^{N(n+1)}=C^{\prime} 2^{N(n+1)}=: D_{n}
\end{array}
$$

for any $n \geq 0$ with $C^{\prime}$ independent of $n$. By setting

$$
b_{n}:=\max \left\{1,\|u\|_{L^{\tilde{p}\left(N+\beta_{n}\right)\left(B\left(x_{0}, R_{n}^{\prime}\right)\right)}}\right\},
$$

and by Lemma 5.2 we obtain

$$
b_{n+1} \leq C^{\frac{1}{N+\beta_{n}}}\left(N+\beta_{n}\right)^{\frac{N}{N+\beta_{n}}}\left(C_{0}+D_{n}\right)^{\frac{1}{N+\beta_{n}}} b_{n}
$$

for every $n \geq 0$ with $C:=2^{N}\left(C_{*}+1\right)^{N} B_{0}$. Put $P:=\tilde{p} N / \bar{p}^{*}<1$. Then, $N+\beta_{n+1}=\left(N+\beta_{n}\right) / P, \beta_{n+1}>\beta_{n} / P>\beta_{0}(1 / P)^{n+1} \rightarrow \infty$ as $n \rightarrow \infty$. Moreover, we see that

$$
\begin{aligned}
& S_{1}:=\sum_{n=0}^{\infty} \frac{1}{N+\beta_{n}}=\frac{1}{N+\beta_{0}} \sum_{n=0}^{\infty} P^{n}=\frac{1}{\left(N+\beta_{0}\right)(1-P)}<\infty, \\
& S_{2}:=\ln \prod_{n=0}^{\infty}\left(N+\beta_{n}\right)^{\frac{N}{N+\beta_{n}}}=\frac{N}{N+\beta_{0}} \sum_{n=0}^{\infty} P^{n}\left(\ln \left(N+\beta_{0}\right)+n \ln P^{-1}\right)<\infty
\end{aligned}
$$

and

$$
\begin{aligned}
S_{3} & :=\ln \prod_{n=0}^{\infty}\left(C_{0}+D_{n}\right)^{\frac{1}{N+\beta_{n}}}=\sum_{n=0}^{\infty} \frac{P^{n}}{N+\beta_{0}} \ln \left(C_{0}+D_{n}\right) \\
& \leq \sum_{n=0}^{\infty} \frac{P^{n}}{N+\beta_{0}} N(n+1) \ln \left(C_{0}+C^{\prime}\right) 2<\infty .
\end{aligned}
$$


As a result, by iteration in (89) and $\tilde{p}\left(N+\beta_{0}\right)=\bar{p}^{*}$, we obtain

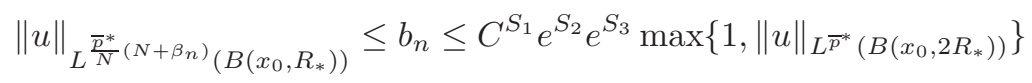

for every $n \geq 1$. Letting $n \rightarrow \infty$, this ensures that

$$
\|u\|_{L^{\infty}\left(B\left(x_{0}, R_{*}\right)\right)} \leq C^{S_{1}} e^{S_{2}} e^{S_{3}} \max \left\{1,\|u\|_{L^{\bar{p}^{*}}\left(B\left(x_{0}, 2 R_{*}\right)\right)}\right\} .
$$

By using the embedding of $W^{1, N}(D)$ to $L^{\bar{p}^{*}}(D),(90)$ yields that

$$
\begin{aligned}
\|u\|_{L^{\infty}\left(B\left(x_{0}, R_{*}\right)\right)} & \leq C^{S_{1}} e^{S_{2}} e^{S_{3}} \max \left\{1,\|u\|_{L^{\bar{p}^{*}}(D)}\right\} \\
& \leq C^{S_{1}} e^{S_{2}} e^{S_{3}} \max \left\{1, C_{*}\|u\|_{W^{1, N}(D)}\right\}
\end{aligned}
$$

whence $u$ is bounded in $D$ because $x_{0} \in D$ is arbitrary and the constant $C^{S_{1}} e^{p S_{2}} e^{S_{3}}$ is independent of $x_{0}$.

\subsection{Proof of Theorem 1.1}

In this section, we denote $B_{n}:=B_{n}(0)$ the open ball centered at the origin with radius $n$. Throughout this section, we will consider $\lambda \in\left(0, \lambda^{*}\right)$ fixed. The space $W^{1, N}\left(B_{n}\right)$ is endowed with the norm

$$
\|u\|_{N, n}^{N}:=\int_{B_{n}}\left(|\nabla u|^{N}+|u|^{N}\right) d x .
$$

By applying Theorem 4.1 with $D=B_{n}(n \in \mathbb{N})$, we obtain a solution $u_{n} \in W_{0}^{1, N}\left(B_{n}\right)$ of the problem

$\left(P_{n}\right) \quad\left\{\begin{array}{lll}-\Delta_{N} u+|u|^{N-2} u=\lambda a(x)|u|^{q-2} u+f(u) & \text { in } & B_{n} \\ u>0 & \text { in } & B_{n} \\ u(x)=0 & \text { on } & \partial B_{n} .\end{array}\right.$ is,

Again, (70) and (80) shows the boundedness of $\left\{u_{n}\right\}_{n>0}$ in $W_{0}^{1, N}(D)$. That is,

$$
\left\|u_{n}\right\|_{N, n} \leq \tilde{\varrho} \text { for all } n \in \mathbb{N}
$$

where

$$
\tilde{\varrho}:=\min \left\{\left(2 \lambda C_{1}\right)^{1 /(N-q)}, \varrho\right\}
$$

is independent of $B_{n}$.

If $n \geq m+1$, notice that

$$
\int_{B_{m+1}}\left(\left|\nabla u_{n}\right|^{N-2} \nabla u_{n} \nabla \varphi+\left|u_{n}\right|^{N-2} u_{n} \varphi-\lambda a(x)\left|u_{n}\right|^{q-2} u_{n} \varphi-f\left(u_{n}\right) \varphi\right) d x=0
$$

for all $\varphi \in C_{0}^{\infty}\left(B_{m+1}\right)$. By (92) we obtain

$$
\left\|u_{n}\right\|_{N, m+1} \leq\left\|u_{n}\right\|_{N, n} \leq \tilde{\varrho}
$$


Therefore, there exists $u_{\lambda} \in W^{1, N}\left(B_{m+1}\right)$ such that

$$
\begin{aligned}
u_{n} & \rightarrow u_{\lambda} \quad \text { in } W^{1, N}\left(B_{m+1}\right), \\
u_{n} & \rightarrow u_{\lambda} \quad \text { in } L^{s}\left(B_{m+1}\right), s \geq N, \\
u_{n}(x) & \rightarrow u_{\lambda}(x) \quad \text { a.e. } x \in B_{m+1},
\end{aligned}
$$

as $n \rightarrow \infty$.

By inequality (91) in Proposition 4 and (94), we infer that

$$
\left\|u_{n}\right\|_{L^{\infty}\left(B_{m+1}\right)} \leq C^{S_{1}} e^{S_{2}} e^{S_{3}} \max \left\{1, C_{*} \tilde{\varrho}\right\}=: \Theta .
$$

Now we use regularity result up to the boundary due to Lieberman [27, Theorem 1.7], to conclude from (98) that

$$
\left\|u_{n}\right\|_{C^{1, \beta}\left(\overline{B_{m}}\right)} \leq \vartheta
$$

where $\beta \in(0,1)$ and $\vartheta$ is independent of $n$. Thus, using (99) and Arzelà-Ascoli theorem, we conclude that

$$
u_{\lambda} \in C^{1, \alpha}\left(\overline{B_{m}}\right) \text { for some } \alpha \in(0, \beta) .
$$

We also have

$$
f\left(u_{n}\right) \rightarrow f\left(u_{\lambda}\right) \text { weakly in } L^{N^{\prime}}\left(B_{m+1}\right),
$$

as $n \rightarrow \infty$. Indeed, notice that

$$
\begin{aligned}
& \int_{B_{m+1}}\left|f\left(u_{n}\right)\right|^{N^{\prime}} d x \leq \int_{B_{m+1}}\left(u_{n}^{(p-1)} \phi_{N}\left(2^{\frac{N}{N-1}} \alpha\left|u_{n}\right|^{\frac{N}{N-1}}\right)\right)^{N^{\prime}} d x \leq
\end{aligned}
$$

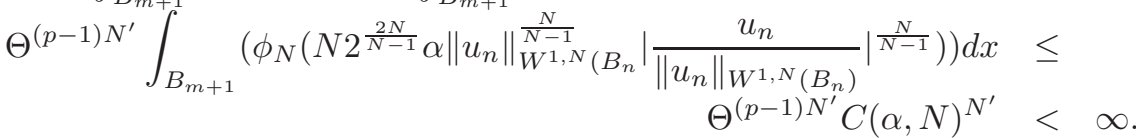

Since

$$
u_{n} \rightarrow u_{\lambda} \text { a.e. in } B_{m+1},
$$

by the continuity of $f$ we have

$$
f\left(u_{n}(\cdot)\right) \rightarrow f\left(u_{\lambda}(\cdot)\right) \text { a.e. in } B_{m+1} .
$$

Hence, [19, Theorem 13.44] leads to

$$
f\left(u_{n}\right) \rightarrow f\left(u_{\lambda}\right) \text { weakly in } L^{N^{\prime}}\left(B_{m+1}\right) .
$$

Let us show that $u_{n}$ converges to $u_{\lambda}$ strongly in $W^{1, N}\left(B_{m}\right)$. To this end, fix $l \in \mathbb{N}$ and choose a smooth function $\psi_{l}$ satisfying $0 \leq \psi_{l} \leq 1, \psi_{l}(r)=1$ if $r \leq m$ and $\psi_{l}(r)=0$ if $r \geq m+1 / l$. Setting $\eta_{l}(x):=\psi_{l}(|x|)$, we note that $\left(u_{n}-u_{\lambda}\right) \eta_{l} \in W_{0}^{1, N}\left(B_{m+1}\right) \subset W_{0}^{1, N}\left(B_{n}\right)$ for any $n \geq m+1$. Denote 


$$
V_{n}=\int_{B_{m}}\left|\nabla u_{n}\right|^{N-2} \nabla u_{n}\left(\nabla u_{n}-\nabla u_{\lambda}\right) d x
$$

Using $\left(u_{n}-u_{\lambda}\right) \eta_{l}$ as test function in (93) and invoking the growth condition (6), we obtain

$$
\begin{aligned}
V_{n}= & \int_{|x|<m+1 / l}\left(\lambda a(x) u_{n}^{q-1}+f\left(u_{n}\right)-u_{n}^{N-1}\right)\left(u_{n}-u_{\lambda}\right) \eta_{l} d x \\
& -\int_{m \leq|x|<m+1 / l}\left|\nabla u_{n}\right|^{N-2} \nabla u_{n} \nabla\left(u_{n}-u_{\lambda}\right) \eta_{l} d x \\
& -\int_{m \leq|x|<m+1 / l}\left|\nabla u_{n}\right|^{N-2} \nabla u_{n} \nabla \eta_{l}\left(u_{n}-u_{\lambda}\right) d x \\
\leq & \int_{B_{m+1}}\left(\lambda a(x) u_{n}^{q-1}+u_{n}^{p-1} \phi_{N}\left(2^{\frac{N}{N-1}} \alpha\left|u_{n}\right|^{\frac{N}{N-1}}\right)+u_{n}^{N-1}\right)\left|u_{n}-u_{\lambda}\right| d x \\
& +\int_{m \leq|x|<m+1 / l}\left|\nabla u_{n}\right|^{N-1}\left|\nabla u_{\lambda}\right| d x \\
& +d_{l} \int_{m \leq|x|<m+1 / l}\left|\nabla u_{n}\right|^{N-1}\left|u_{n}-u_{\lambda}\right| d x \\
\equiv & I_{n}^{1}+I_{n}^{2}+I_{n}^{3},
\end{aligned}
$$

where $d_{l}:=\sup _{|x|<m+1 / l}\left|\nabla \eta_{l}(x)\right|$.

By Hölder's inequality and (92) we have

$$
\begin{aligned}
I_{n}^{1} \leq & \left\|u_{n}-u_{\lambda}\right\|_{L^{N}\left(B_{m+1}\right)}\left\{\lambda\|a\|_{L^{N /(N-q)}\left(B_{m+1}\right)}\left\|u_{n}\right\|_{W^{1, N}\left(B_{m}+1\right)}^{q}\right. \\
& \left.+\vartheta^{p-1} C(\alpha, N)+\left\|u_{n}\right\|_{W^{1, N}\left(B_{m}+1\right)}^{N-1}\right\} \\
\leq & \bar{C}\left\|u_{n}-u_{\lambda}\right\|_{L^{N}\left(B_{m+1}\right)}
\end{aligned}
$$

where $\bar{C}$ is a positive constant independent of $u_{n}, n, m$ and $l$.

By Hölder's inequality, the following estimates follow:

$$
\begin{gathered}
I_{n}^{2} \leq\left\|u_{n}\right\|_{W^{1, N}\left(B_{m+1}\right)}^{N-1}\left(\int_{m \leq|x|<m+1 / l}\left|\nabla u_{\lambda}\right|^{N} d x\right)^{1 / N}, \\
I_{n}^{3} \leq d_{l}\left\|u_{n}-u_{\lambda}\right\|_{L^{N}\left(B_{m+1}\right)}\left\|\nabla u_{n}\right\|_{L^{N}\left(B_{m+1}\right)}^{N-1} .
\end{gathered}
$$

Thereby, from (92) and (96) we derive

$$
\limsup _{n \rightarrow \infty} V_{n} \leq \tilde{\varrho}^{N-1}\left(\int_{m \leq|x|<m+1 / l}\left|\nabla u_{\lambda}\right|^{N} d x\right)^{1 / N},
$$


for all $l \in \mathbb{N}$. Thus, letting $l \rightarrow \infty$, we obtain

$$
\limsup _{n \rightarrow \infty} V_{n} \leq 0
$$

As known from $(95), u_{n}$ weakly converges to $u_{\lambda}$ in $W^{1, N}\left(B_{m}\right)$, so we may write

$$
\begin{aligned}
V_{n}+o(1) & =\int_{B_{m}}\left(\left|\nabla u_{n}\right|^{N-2} \nabla u_{n}-\left|\nabla u_{\lambda}\right|^{N-2} \nabla u_{\lambda}\right)\left(\nabla u_{n}-\nabla u_{\lambda}\right) d x \\
& \geq\left(\left\|\nabla u_{n}\right\|_{L^{N}\left(B_{m}\right)}^{N-1}-\left\|\nabla u_{\lambda}\right\|_{L^{N}\left(B_{m}\right)}^{N-1}\right)\left(\left\|\nabla u_{n}\right\|_{L^{N}\left(B_{m}\right)}-\left\|\nabla u_{\lambda}\right\|_{L^{N}\left(B_{m}\right)}\right) \\
& \geq 0 .
\end{aligned}
$$

By (105) and (106), we obtain $\lim _{n \rightarrow \infty} V_{n}=0, \lim _{n \rightarrow \infty}\left\|\nabla u_{n}\right\|_{L^{N}\left(B_{m}\right)}=\left\|\nabla u_{\lambda}\right\|_{L^{N}\left(B_{m}\right)}$. This implies that $u_{n}$ converges to $u_{\lambda}$ strongly in $W^{1, N}\left(B_{m}\right)$ because the spaces $W^{1, N}\left(B_{m}\right)$ is uniformly convex.

Now, since $u_{n}>0$ in $B_{m}$, we infer that $u_{\lambda}$ is a nonnegative solution of the problem

$$
-\Delta_{N} u_{\lambda}+u_{\lambda}^{N-1}=\lambda a(x) u_{\lambda}^{q-1}+f\left(u_{\lambda}\right) \quad \text { in } B_{m}, \quad u_{\lambda} \geq 0 \quad \text { on } \partial B_{m} .
$$

By using (24) and since $C_{0}^{\infty}\left(\mathbb{R}^{N}\right)$ is dense in $W^{1, N}\left(\mathbb{R}^{N}\right)$, by a diagonal argument, there exist a relabeled subsequence of $\left\{\tilde{u}_{n}\right\}$ and a function $u_{\lambda} \in$ $W^{1, N}\left(\mathbb{R}^{N}\right)$ such that

$$
\begin{gathered}
\tilde{u}_{n} \rightarrow u_{\lambda} \text { in } W^{1, N}\left(\mathbb{R}^{N}\right), \\
\tilde{u}_{n}(x) \rightarrow u_{\lambda}(x) \text { for a.e } x \in \mathbb{R}^{N} .
\end{gathered}
$$

These convergence properties, and some iteration process, ensure that $u_{\lambda}$ is a solution of problem (5), that belongs to $C_{l o c}^{1}\left(\mathbb{R}^{N}\right)$ (see (100)).

The next step in the proof is to show that $u_{\lambda}$ does not vanish in $\mathbb{R}^{N}$. Indeed, Lemma 2.2 provides a solution $u_{\lambda, m}$ of the problem

$$
\left\{\begin{array}{lll}
-\Delta_{N} u+|u|^{N-2} u=\lambda a_{m}|u|^{q-2} u & \text { in } & B_{m} \\
u>0 & \text { in } & B_{m} \\
u(x)=0 & \text { on } \partial B_{m}
\end{array}\right.
$$

where

$$
a_{m}=\inf _{B_{m}} a(x) .
$$

Since $\lambda a(x) t^{q-1}+f(t) \geq \lambda a_{m} t^{q-1}$ for all $x \in \mathbb{R}^{N}$. We are thus in a position to apply Proposition 2 to the functions $u_{\lambda, m}$ and $\tilde{u}_{n}$ with $n>m$, in place of $u_{1}=u_{\lambda, m}$ and $u_{2}=\tilde{u}_{n}$, respectively, which renders $\tilde{u}_{n} \geq u_{\lambda, m}$ in $B_{m}$ for every $n>m$. This enables us to deduce that $u_{\lambda} \geq u_{\lambda, m}$ in $B_{m}$, so $u_{\lambda}(x)>0$ for every $x \in \mathbb{R}^{N}$, because $m$ was arbitrary chosen.

Furthermore, since $\tilde{u}_{n}$ weakly converges to $u_{\lambda}$ in $W^{1, N}\left(\mathbb{R}^{N}\right)$, by (94) we have

$$
\left\|u_{\lambda}\right\|_{W^{1, N}\left(\mathbb{R}^{N}\right)} \leq \liminf _{n \rightarrow \infty}\left\|\tilde{u}_{n}\right\|_{W^{1, N}\left(\mathbb{R}^{N}\right)}=\liminf _{n \rightarrow \infty}\left\|\tilde{u}_{n}\right\|_{N, n} \leq \tilde{\varrho}
$$


and then, we obtain $u_{\lambda} \in W^{1, N}\left(\mathbb{R}^{N}\right)$, and

$$
\left\|u_{\lambda}\right\|_{W^{1, N}\left(\mathbb{R}^{N}\right)} \rightarrow 0, \text { as } \lambda \rightarrow 0 .
$$

Thus, the proof of Theorem 1.1 is complete.

\subsection{Proof of Proposition 1}

In this proof, we borrowed some ideas from [6]. For the sake of contradiction, suppose that $\lambda^{*}=\infty$. Thus, there is a sequence $\lambda_{n} \rightarrow \infty$ and corresponding solutions $u_{\lambda_{n}}>0$ in $\mathbb{R}^{N}$ given by Theorem 1.1. In what follows, define $a_{R}=$ $\inf _{B_{R}(0)} a(r)$, for some $R>0$ fixed. Define also

$$
\mathcal{P}(x, t)=\lambda a(x) t^{q-1}+t^{p-1} \phi_{N}\left(\alpha|t|^{\frac{N}{N-1}}\right)
$$

and

$$
P_{1}(t)=\Lambda t^{q-1}+t^{p-1} \phi_{N}\left(\alpha|t|^{\frac{N}{N-1}}\right)
$$

where $\Lambda=\lambda a_{R}$.

We claim that there exists a constant $C_{\Lambda}>0$ such that

$$
P(x, t) \geq C_{\Lambda} t^{N-1} \text { for every } t>0 .
$$

Indeed, define the function $Q(t)=P_{1}(t) t^{-(N-1)}$. Then, since $q<N<p$, $Q(t) \rightarrow \infty$ as $t \rightarrow 0^{+}$and as $t \rightarrow \infty$. The minimum value is $Q\left(t_{1}\right)=C_{\Lambda}$, where $t_{1}>0$ is the unique solution of

$$
\phi_{N}\left(\alpha t^{\frac{N}{N-1}}\right)(p-N) t^{p-q}+\alpha \frac{N}{N-1} t^{\frac{(N-1)(p-q+1)+1}{N-1}} \phi_{N-1}\left(\alpha t^{\frac{N}{N-1}}\right)=\Lambda(N-q) .
$$

Indeed, let us define

$$
H(t):=\phi_{N}\left(\alpha t^{\frac{N}{N-1}}\right)(p-N) t^{p-q}+\alpha \frac{N}{N-1} t^{\frac{(N-1)(p-q+1)+1}{N-1}} \phi_{N-1}\left(\alpha t^{\frac{N}{N-1}}\right) .
$$

Note that $H(0)=0$ and $H(t) \rightarrow \infty$ as $t \rightarrow \infty$. Since

$$
\begin{aligned}
H^{\prime}(t) & =(p-N)(p-q) t^{p-q-1} \phi_{N}\left(\alpha t^{\frac{N}{N-1}}\right) \\
& +\frac{\alpha N(p-N)}{N-1} t^{p-q+\frac{1}{N}} \phi_{N-1}\left(\alpha t^{\frac{N}{N-1}}\right) \\
& +\frac{N \alpha}{N-1}\left[\frac{(N-1)(p-q+1)+1}{N-1}\right] t^{\frac{(N-1)(p-q+1)+1}{N-1}-1} \phi_{N-1}\left(\alpha t^{\frac{N}{N-1}}\right) \\
& +\frac{\alpha^{2} N^{2}}{(N-1)^{2}} t^{\frac{(N-1)(p-q+1)+1}{N-1}+\frac{1}{N-1}} \phi_{N-2}\left(\alpha t^{\frac{N}{N-1}}\right)>0, \quad \forall t \geq 0,
\end{aligned}
$$

hence $H$ is increasing. Therefore, there is a unique point $t_{1}>0$ such that $H\left(t_{1}\right)=\Lambda(N-q)$. Consequently, by the arguments before, $t_{1}>0$ is the unique point of minimum of $Q$, with $Q\left(t_{1}\right)=C_{\Lambda}$. 
Denote by $\sigma_{1}>0$ and $\varphi_{1}>0$, respectively, the principal eigenvalue and corresponding eigenfunction of the eigenvalue problem

$$
\left\{\begin{array}{lll}
-\Delta_{N}\left(\varphi_{1}\right)=\sigma_{1}\left|\varphi_{1}\right|^{N-2} \varphi_{1} & \text { in } & B_{R}(0) \\
\varphi_{1}=0 & \text { on } & \partial B_{R}(0)
\end{array}\right.
$$

Since $C_{\Lambda}$ increases as $\lambda_{n}$ increases, for $\delta>0$ small enough, there is $\lambda_{0}$ in this sequence such that the corresponding constant satisfies $C_{\Lambda_{0}} \geq \sigma_{1}+\delta+1$. Hence the solution $u_{\lambda_{0}}$ of (5) associated to $\lambda_{0}$ satisfies $u_{\lambda_{0}}>0$ in $\mathbb{R}^{N}$ and

$$
\begin{cases}-\Delta_{N} u_{\lambda_{0}} \geq\left(C_{\Lambda_{0}}-1\right)\left|u_{\lambda_{0}}\right|^{N-2} u_{\lambda_{0}} \geq\left(\sigma_{1}+\delta\right)\left|u_{\lambda_{0}}\right|^{N-2} u_{\lambda_{0}} & \text { in } \quad B_{R}(0) \\ u_{\lambda_{0}} \geq 0 & \text { on } \partial B_{R}(0) .\end{cases}
$$

Otherwise, taking $\varepsilon>0$ small enough we obtain $\varepsilon \varphi_{1}<u_{\lambda_{0}}$ in $B_{R}(0)$ and

$$
\left\{\begin{array}{lll}
-\Delta_{N}\left(\varepsilon \varphi_{1}\right)=\sigma_{1}\left|\varepsilon \varphi_{1}\right|^{N-2}\left(\varepsilon \varphi_{1}\right) \leq\left(\sigma_{1}+\delta\right)\left|\varepsilon \varphi_{1}\right|^{N-2}\left(\varepsilon \varphi_{1}\right) & \text { in } & B_{R}(0) \\
\varphi_{1}=0 & \text { on } & \partial B_{R}(0) .
\end{array}\right.
$$

By the method of subsolution and supersolution, see [6, Theorem 2.1], there is a solution $\varepsilon \varphi_{1} \leq \omega \leq u_{\lambda_{0}}$ in $B_{R}(0)$ of

$$
\left\{\begin{array}{lll}
-\Delta_{N} \omega=\left(\sigma_{1}+\delta\right)|\omega|^{N-2} \omega & \text { in } & B_{R}(0) \\
\omega=0 & \text { on } & \partial B_{R}(0) .
\end{array}\right.
$$

Hence there is a contradiction to the fact that $\sigma_{1}$ is isolated (see [28]). Therefore, $\lambda^{*}<\infty$.

\subsection{Proof of Theorem 1.2}

By using the same arguments as in the proof of Theorem 1.1, with $D=B_{n}$, and considering (107), we can pass to the limit $n \rightarrow+\infty$ in (91) to obtain

$$
\left\|u_{\lambda}\right\|_{L^{\infty}\left(\mathbb{R}^{N}\right)} \leq C^{S_{1}} e^{S_{2}} e^{S_{3}} \max \left\{1, C_{*} \tilde{\varrho}\right\} .
$$

Since $u_{\lambda}$ is bounded in $\mathbb{R}^{N}$, we put $M_{0}:=\left\|u_{\lambda}\right\|_{L^{\infty}\left(\mathbb{R}^{N}\right)}$. Then, by Lemma 5.1, we have

$$
\begin{aligned}
& \int_{B\left(x_{0}, R^{\prime}\right)} f\left(u_{\lambda}\right) u_{\lambda}\left(u_{\lambda}\right)_{M}^{\beta} \eta^{N} d x \leq C(\varrho)\left\|u_{\lambda}\right\|_{L^{\tilde{p}(N+\beta)}\left(B\left(x_{0}, R^{\prime}\right)\right)}^{\beta} B_{R^{\prime}} \\
& \int_{B\left(x_{0}, R^{\prime}\right)} a(x) u_{\lambda}^{q}\left(u_{\lambda}\right)_{M}^{\beta} \eta^{N} d x \leq\|a\|_{L^{\gamma_{1}}\left(B\left(x_{0}, R^{\prime}\right)\right)} M_{0}^{q}\left\|u_{\lambda}\right\|_{L^{\tilde{p}(N+\beta)}\left(B\left(x_{0}, R^{\prime}\right)\right)}^{\beta} B_{R^{\prime}}
\end{aligned}
$$

By Hölder inequality, we obtain

$$
\left\|u_{\lambda}\right\|_{L^{\tilde{p}(N+\beta)}\left(B\left(x_{0}, R^{\prime}\right)\right)}^{N+\beta} \leq M_{0}^{N}\left\|u_{\lambda}\right\|_{L^{\tilde{p}(N+\beta)}\left(B\left(x_{0}, R^{\prime}\right)\right)}^{\beta} B_{R^{\prime}}
$$


Now, fix $x_{0} \in \mathbb{R}^{N}$. It follows from the argument as in the proof of Lemma 5.2 with (110), (111) and (112) that

$$
\begin{aligned}
\left\|u_{\lambda}\right\|^{N+\beta} & L^{\frac{\bar{p}^{*}}{N}(N+\beta)}\left(B\left(x_{0}, R\right)\right) \\
& \leq 2^{N}(N+\beta)^{N} C_{*}^{N} B_{R^{\prime}}\left(C_{R^{\prime}}+D_{R, R^{\prime}}\right)\left(M_{0}+1\right)^{N}\left\|u_{\lambda}\right\|_{L^{\tilde{p}(N+\beta)}\left(B\left(x_{0}, R^{\prime}\right)\right)}^{\beta}
\end{aligned}
$$

provided $u_{\lambda} \in L^{\tilde{p}(N+\beta)}\left(B\left(x_{0}, R^{\prime}\right)\right)$. Choose $\gamma_{1}, \tilde{p}$ and define sequences $\left\{\beta_{n}\right\}$, $\left\{R_{n}^{\prime}\right\}$ and $\left\{R_{n}\right\}$ as in the proof of Proposition 4. Set

$$
V_{n}:=\left\|u_{\lambda}\right\|_{L^{\tilde{p}\left(N+\beta_{n}\right)}\left(B\left(x_{0}, R_{n}^{\prime}\right)\right)}^{\beta_{n}} .
$$

The remainder of the proof follows with the same arguments as in in the proof of $[15$, Theorem 2] to conclude that

$$
V_{n}^{\frac{N+\beta_{n-1}}{\beta_{n}}} \leq C\left(N+\beta_{n-1}\right)^{N}\left(C_{0}+D_{n-1}\right) V_{n-1}
$$

with $C:=2^{N} C_{*}^{N} B_{0}\left(M_{0}+1\right)^{N}$. Recall that

$$
\beta_{n}+N=P^{-1}\left(N+\beta_{n-1}\right) \text { and } \frac{N}{N+\beta_{0}}=P .
$$

Define

$$
Q_{n}:=\prod_{k=2}^{n+1}\left(1+\frac{P^{k}}{1-P^{k}}\right)=\prod_{k=2}^{n+1}\left(1-P^{k}\right)^{-1} \quad \text { and } \quad W_{n}:=\left(C_{0}+D_{n}\right) .
$$

Then, the inequality (114) leads to

$$
\begin{aligned}
\ln V_{n} \leq & \frac{\beta_{n}}{N+\beta_{n-1}}\left(\ln V_{n-1}+\ln \left[C\left(N+\beta_{n-1}\right)^{N}\right]+\ln W_{n-1}\right) \\
= & P^{-1}\left(1-P^{n+1}\right)\left(\ln V_{n-1}+N \ln \left[C P^{-n+1}\left(N+\beta_{0}\right)\right]+\ln W_{n-1}\right) \\
\leq & P^{-1}\left(1-P^{n+1}\right) \ln V_{n-1}+N P^{-1} \ln \left[(C+1) P^{-n+1}\left(N+\beta_{0}\right)\right]+P^{-1} \ln W_{n-1} \\
\leq & P^{-n}\left(\prod_{k=1}^{n}\left(1-P^{k+1}\right)\right) \ln V_{0}+N \sum_{k=1}^{n} P^{-k} \ln \left[(C+1) P^{-n+k}\left(N+\beta_{0}\right)\right] \\
& \quad+\sum_{k=1}^{n} P^{-k} \ln W_{n-k} \\
& =P^{-n} Q_{n}^{-1} \ln V_{0}+N \sum_{k=1}^{n} P^{-k} \ln \left[(C+1) P^{-n+k}\left(N+\beta_{0}\right)\right]+\sum_{k=1}^{n} P^{-k} \ln W_{n-k}
\end{aligned}
$$

for every $n$ because of $\ln \left[(C+1) P^{-n+1}\left(N+\beta_{0}\right)\right]>0$ and $\ln W_{n}>0$ for all $n$. Therefore, we have

$$
\begin{aligned}
& \ln \left\|u_{\lambda}\right\|_{L^{\tilde{p}\left(N+\beta_{n}\right)}\left(B\left(x_{0}, R_{n}^{\prime}\right)\right)}=\frac{\ln V_{n}}{\alpha_{n}}=\frac{P^{n} \ln V_{n}}{N+\beta_{0}-N P^{n}} \\
& \leq \frac{Q_{n}^{-1} \ln V_{0}}{N+\beta_{0}-N P^{n}}+\frac{\sum_{l=0}^{n-1} P^{l} \ln \left[(C+1) P^{-l}\left(N+\beta_{0}\right)\right]}{N+\beta_{0}-N P^{n}}+\frac{\sum_{l=0}^{n-1} P^{l} \ln W_{l}}{N+\beta_{0}-N P^{n}} .
\end{aligned}
$$


Here, taking a sufficiently large positive constant $C^{\prime}$ independent of $n$, we see that

$$
\sum_{l=0}^{n-1} P^{l} \ln \left[(C+1) P^{-l}\left(N+\beta_{0}\right)\right] \leq C^{\prime} \sum_{l=0}^{\infty} P^{l}(l+1)=: S_{1}<\infty
$$

and

$$
\sum_{l=0}^{n-1} P^{l} \ln W_{l} \leq C^{\prime} \sum_{l=0}^{n-1} P^{l}(l+1) \leq C^{\prime} \sum_{l=0}^{\infty} P^{l}(l+1)=: S_{2}<\infty .
$$

Next, we shall show that $\left\{Q_{n}\right\}$ is a convergent sequence. It is easily see that $\left\{Q_{n}\right\}$ is increasing. Moreover, setting $d_{k}:=\ln \left(1+\frac{P^{k}}{1-P^{k}}\right)$, we see that

$$
\lim _{k \rightarrow \infty} \frac{d_{k+1}}{d_{k}}=\lim _{k \rightarrow \infty} \frac{\ln \left(1-P^{k+1}\right)}{\ln \left(1-P^{k}\right)}=\lim _{k \rightarrow \infty} \frac{1-P^{k}}{1-P^{k+1}} P=P<1
$$

by L'Hospital's rule. This implies that

$$
\ln Q_{n}=\sum_{k=2}^{n+1} \ln \left(1+\frac{P^{k}}{1-P^{k}}\right) \leq \sum_{k=1}^{\infty} \ln \left(1+\frac{P^{k}}{1-P^{k}}\right)<\infty .
$$

Therefore, $\left\{Q_{n}\right\}$ is bounded from above, whence $\left\{Q_{n}\right\}$ converges and

$$
1<\frac{1}{1-P^{2}}=Q_{1} \leq Q_{\infty}:=\lim _{n \rightarrow \infty} Q_{n}<\infty
$$

holds. Consequently, letting $n \rightarrow \infty$ in (115), we have

$$
\left\|u_{\lambda}\right\|_{L^{\infty}\left(B\left(x_{0}, R_{*}\right)\right)} \leq\left(N S_{1} S_{2}\right)^{\frac{1}{N+\beta_{0}}}\left\|u_{\lambda}\right\|_{\left.L^{\bar{p}^{*}}\left(B\left(x_{0}\right), 2 R_{*}\right)\right)}^{\frac{\beta_{0}}{\left(\beta_{\infty}\right)}} .
$$

This yields our conclusion since $\left\|u_{\lambda}\right\|_{L_{\bar{p}^{*}}\left(B\left(x_{0}, 2 R_{*}\right)\right)} \rightarrow 0$ as $\left|x_{0}\right| \rightarrow \infty, \beta_{0}>0$ and the constant $N S_{1} S_{2}$ is independent of $x_{0}$.

\subsection{Proof of Corollary 1}

Notice that by (109), $\left\|u_{\lambda}\right\|_{L^{\infty}\left(\mathbb{R}^{N}\right)}$ is uniformly bounded in the variable $\lambda$. Hence, the constant $C$ in (114) is uniformly bounded in the variable $\lambda$.

By (116), we obtain

$$
\left\|u_{\lambda}\right\|_{L^{\infty}\left(B\left(x_{0}, R_{*}\right)\right)} \leq\left(N S_{1} S_{2}\right)^{\frac{1}{N+\beta_{0}}}\left\|u_{\lambda}\right\|_{L^{\bar{p}^{*}}\left(\mathbb{R}^{N}\right)}^{\frac{\beta_{0}}{\left(N+\beta_{0}\right)}} \leq \tilde{C}\left\|u_{\lambda}\right\|_{W^{1, N}\left(\mathbb{R}^{N}\right)}^{\frac{\beta_{0}}{\left(N+Q_{\infty}\right)}},
$$

with $\tilde{C}$ independent of $\lambda$. Since $x_{0}$ is arbitrary, on has

$$
\left\|u_{\lambda}\right\|_{L^{\infty}\left(\mathbb{R}^{N}\right)} \leq \tilde{C}\left\|u_{\lambda}\right\|_{W^{1, N}\left(\mathbb{R}^{N}\right)}^{\frac{\beta_{0}}{\left(N+\beta_{0}\right) Q_{\infty}}} .
$$

Thus, (108) ensure that

$$
\left\|u_{\lambda}\right\|_{L^{\infty}\left(\mathbb{R}^{N}\right)} \rightarrow 0
$$

as $\lambda \rightarrow 0$. 


\section{Acknowledgements}

L. F.O Faria was partially supported by FAPEMIG CEX APQ 02374/17. A.L.A.

de Araujo was partially supported by FAPEMIG FORTIS-10254/2014 and CNPQ.

\section{References}

[1] C.O. Alves, L.R. de Freitas, Multiplicity of nonradial solutions for a class of quasilinear equations on annulus with exponential critical growth, Topol. Methods Nonlinear Anal. 39 (2012) 243-262.

[2] C. O. Alves, M. A. S. Souto, M. Montenegro, Existence of a ground state solution for a nonlinear scalar field equation with critical growth, Calc. Var. (2012) 43:537-554.

[3] D. Ander, G. Derrick, Stability of time dependent particle like solutions in nonlinear field theories. J. Math. Phys. 11, 1336-1346 (1970); and 12, 945-952 (1971).

[4] Aouaoui, S. Existence result for some elliptic quasilinear equation involving the $N$-Laplacian in $\mathbb{R}^{N}$ with a large class of nonlinearities. Ricerche Mat. 67 , 875-889 (2018).

[5] H. Brézis, Functional Analysis, Sobolev Spaces and Partial Differential Equations, 585 DOI 10.1007/978-0-387-70914-7, Springer Science+Business Media, LLC 2011.

[6] P. C. Carrião, J. V. Gonçalves and O. H. Miyagaki, Existence and nonexistence in a class of equations with supercritical growth. Applicable Analysis. 74 (2000) 275-287.

[7] T. Cazenave, Equations de Schrödinger nonlineaires en dimension deux., Proc Roy Soc Edinburgh Sect A, 1979, 84(3-4): 327-346.

[8] S. Coleman, The fate of the false vacuum. I-Semi-classical Theory. Phys. Rev. D. 15, 2929 (1977).

[9] Costa, D.G., On a class of elliptic systems in RN . Electron. J. Diff. Eqs. 07-14 (1994).

[10] A. L. A. de Araujo, L. F. O. Faria, Positive solutions of quasilinear elliptic equations with exponential nonlinearity combined with convection term, J. Differential Equations 267 (2019), 4589-4608.

[11] L. R. de Freitas, Multiplicity of solutions for a class of quasilinear equations with exponential critical growth, Nonlinear Analysis 95 (2014) 607-624.

[12] do Ó J.M.: $N$-Laplacian equations in $\mathbb{R}^{N}$ with critical growth. Abstr. Appl. Anal. 2, 301-315 (1997). 
[13] J. M. do Ó, E. Medeiros, U. Severo, On a quasilinear nonhomogeneous elliptic equation with critical growth in $\mathbb{R}^{N}$, J. Differential Equations 246 (4) (2009) 1363-1386.

[14] J. M. do Ó, P. K. Mishra, A. Moameni, Super critical problems with concave and convex nonlinearities in $\mathbb{R}^{N}$, Communications in Contemporary Mathematics, (2020). doi: 10.1142/S0219199720500522.

[15] L.F. Faria, O.H. Miyagaki, M. Tanaka, Existence of a positive solution for problems with $(p, q)$-Laplacian and convection term in $\mathbb{R}^{N}$. Bound Value Probl 2016, 158 (2016).

[16] P. H. Frampton, Consequences of vacuum instability in quantum field theory. Phys. Rev. D. 15 (10), 2922-2928 (1977).

[17] S. Fučík, O. John and J. Nečas, On the existence of Schauder bases in Sobolev spaces, Comment. Math. Univ. Carolinae 13, 163-175 (1972).

[18] B. Gidas, Bifurcation phenomena in mathematical physics and related topics. (1980), Bardos, C., \& Bessis D., editors. Dordrecht, Holland: Reidel.

[19] E. Hewitt and K. Stromberg, Real and Abstract Analysis. Springer-Verlag, 1975 .

[20] F. Isaia, Superposition operators between Sobolev spaces and a nonexistence result of higher-order regular solutions for the p-Laplacian. Nonlinear Anal. 117(10), 87-98 (2015)

[21] S. Kesavan, Topics in functional analysis and applications, John Wiley \& Sons (1989).

[22] O. A. Ladyzhenskaja and N. N. Ural'tseva, Linear and quasilinear elliptic equations, Academic Press, New York-London (1968).

[23] J.F. Lam, B. Lippman, F. Trappert, Self trapped laser beams in plasma. Phys Fluid, 20 1176-1179 (1997).

[24] N. Lam and G. Lu, Existence and multiplicity of solutions to equations of $N$-Laplacian type with critical exponential growth in $\mathbb{R}^{N}$. J. Funct. Anal. 262, 1132-1165 (2012).

[25] Y.X. Li, B. Ruf, A sharp Moser-Trudinger type inequality for unbounded domains in Rn, Indiana Univ. Math. J. 57(1) (2008) 451-480.

[26] G. M. Lieberman, Boundary regularity for solutions of degenerate elliptic equations, Nonlinear Anal. 12, 1203-1219 (1988).

[27] G. M. Lieberman, The natural generalization of the natural conditions of Ladyzhenskaya and Ural'tseva for elliptic equations, Comm. Partial Differential Equations 16, 311-361 (1991). 
[28] P. Lindqvist, On the Equation $\operatorname{div}\left(|\nabla u|^{p-2} \nabla u\right)+\Lambda|u|^{p-2} u=0$. Proceedings of the American Mathematical Society, vol. 109, no. 1, 157-164 (1990).

[29] N. Masmoudi, F. Sani, Trudinger-Moser Inequalities with the Exact Growth Condition in $\mathbb{R}^{N}$ and Applications, Communications in Partial Differential Equations, (40) 1408-1440, 2015.

[30] J. Moser, A sharp form of an inequality by Trudinger. Indiana Univ. Math. J. 20 1077-1092, 1971.

[31] D. Motreanu, V. V. Motreanu and N. S. Papapgeorgou, Multiple constant sign and nodal solutions for nonlinear Neumann eigenvalue problems, Ann. Sc. Norm. Sup. Pisa Cl. Sci. (5) 10, 729-755 (2011).

[32] P. Pucci and J. Serrin, The maximum principle, Progress in Nonlinear Differential Equations and their Applications, 73, Birkhauser Verlag, Basel (2007).

[33] B. Ruf, A sharp Trudinger-Moser type inequality for unbounded domains in $\mathbb{R}^{2}$, J. Funct. Anal. 219 (2005), 340-367.

[34] W. A. Strauss, On weak solutions of semilinear hyperbolic equations, An. Acad. Brasil. Ciênc. 42 645-651 (1970)

[35] S. I. Pohožaev, The Sobolev embedding in the case $p l=n$. Proc. of the Technical Scientific Conference on Advances of Scientific Research 1964-1965, Mathematics Section, (Moskov. Energet. Inst., Moscow), pages 158-170, 1965.

[36] N. S. Trudinger, On imbeddings into Orlicz spaces and some applications. J. Math. Mech. 17 473-483, 1967. 181

[37] Y. Yang, Existence of positive solutions to quasi-linear elliptic equations with exponential growth in the whole Euclidean space. J. Funct. Anal. 262: (2012) 1679-1704.

[38] V.I. Yudovič, Some estimates connected with integral operators and with solutions of elliptic equations. Dokl. Akad. Nauk SSSR. 138 805-808, 1961. 